\title{
Virtual Reality Training and Assessment in Laparoscopic Rectum Surgery
}

\author{
Jun J. Pan ${ }^{1}$, Jian Chang ${ }^{2}$, Xiaosong Yang ${ }^{2}$, Hui Liang ${ }^{5}$, Jian J. Zhang ${ }^{2}$ \\ Tahseen Qureshi ${ }^{3}$, Robert Howell ${ }^{4}$ Tamas Hickish $^{4}$ \\ ${ }^{1}$ Center for Modeling, Simulation and Imaging in Medicine, School of Engineering, \\ Rensselaer Polytechnic Institute, Troy, NY, USA \\ E-mail: pan_junjun@hotmail.com \\ 2 \\ 2 National Centre for Computer Animation, Media School, \\ Bournemouth University, Poole, UK \\ E-mail: \{jchang, xyang, jzhang\}@bournemouth.ac.uk \\ ${ }^{3}$ Poole Hospital, Poole, UK \\ E-mail: taz.qureshi@poole.nhs.uk \\ The Royal Bournemouth and Christchurch Hospitals, Bournemouth, UK \\ E-mail: \{robert.howell, tamas.hickish\}@rbch.nhs.uk \\ ${ }^{5}$ School of Animation \\ Communication University of China, Beijing, China \\ E-mail: hliang@cuc.edu.cn
}

\begin{abstract}
Background Virtual-Reality based simulation techniques offer an efficient and low cost alternative to the conventional surgery training. This article describes a VR training and assessment system in laparoscopic rectum surgery.
\end{abstract}

Methods To give a realistic visual performance of interaction between membrane tissue and surgery tools, a generalized cylinder based collision detection and a multi-layer mass-spring model are presented. A dynamic assessment model is also designed for the hierarchy training evaluation.

Results With this simulator, trainees can operate on the virtual rectum haptically and watch the deformation of soft tissue simultaneously. The system also offers surgeons instructions in real time when improper manipulation happens. Finally, ten subjects tested and evaluated our simulator through questionnaire.

Conclusions This prototype system has been verified by colorectal surgeons through pilot study. They believe the visual performance and the tactile feedback are realistic. It could transfer surgical skills to novices effectively and influence their learning curve.

Keywords collision detection, dissection simulation, evaluation, laparoscopic rectum surgery, dynamic assessment

\section{Introduction}

In late years, the development of minimally invasive surgery (MIS) techniques in clinic, such as laparoscopic and endoscopic surgeries, is remarkable. Compared with traditional open surgery, due to the different eye-hand coordination and the fulcrum effect of the abdominal wall in instrument manipulation [1], performance of MIS requires a special set of psychomotor and perceptual skills. Therefore, laparoscopic surgery is difficult to learn by observation and practice alone, with the conventional apprenticeship concept. A few approaches can be used to influence the learning curves of laparoscopic surgeons. One of them is called training box [2, 3]. These boxes may contain inanimate objects, which just provide mundane tasks. And these training objects are usually made from rubber or silica gel, which lack the realistic feel when handling human tissues. Animal tissue can be used as alternative, but it 
is different from the human anatomy structure. Though cadavers are available, they are quite expensive and hardly provide repetitive practice. In reality, residents in many nations, including the UK, actually develop their laparoscopic surgical skills by directly operating on real patients, under the supervision from senior surgeon [4]. Recently, with the rapid growth of computer power, virtual reality (VR) simulator has become a viable alternative approach, which allows the trainee to hone their skills by operating on virtual patients in a safe and repetitive manner.

The aim of this paper is to describe a VR-based training and assessment system for the laparoscopic rectum surgery. At present, colorectal cancer is the third commonest cancer in the UK, with approximately 40,000 new cases diagnosed annually [4]. It is estimated that $90 \%$ cases are suitable for the laparoscopic resection surgery. However, due to the complicated and technically challenging nature of the laparoscopic colorectal cancer surgery, there is a relative lack of trained surgeons to perform such high demanding operations. On the other hand, although there are several commercial VR based laparoscopic surgery simulators (LapSim [5], Lap Mentor [6], Mentice [7]) in the market, most of them aim to simulate procedures in cholecystectomy (gallbladder removal), ventral hernia repair, ectopic pregnancy and myomectomy. The application in rectal resection surgery for cancer is seldom due to the narrow pelvis, substantial large soft tissue deformation and complex interactions between surgery tools and soft tissue in operation. Therefore, it brings a greater challenge for the haptic response and realistic graphic computation.

In the recent years, a large amount of research has been made in virtual reality based surgery simulation. Pan et al. [8] described a simple simulator for the virtual laparoscopic rectum surgery. They select triangle mesh rather than tetrahedra model to represent the bowl. To meet the requirement of real time graphic performance, a cosserat rod model had been introduced to handle the physical deformation and collision detection. Choi et al. [9] designed a VR training system for laparoscopic oesophagus surgery simulation. They used boundary element method (BEM) to compute the deformation at the assumption of linear response. The mechanical parameters were collected from the experiments of animal tissue and integrated with haptic device. Heng et al. [10] developed a VR simulation system for the knee arthroscopic surgery. In the system, they used the virtual models from the database of Visible Human. And the finite element analysis (FEA) was applied to simulate the deformation and incision of soft tissue in real-time. Haptic rendering [11, 12, 13] is another important research field in VR based surgery simulation. Maciel et al. [14] presented a dynamic point algorithm for line based collision detection in haptics. They simplified the surgical instrument as a section of line with two end points, and a dynamic point is located between the two end points. In this approach, the dynamic point is the closest point on the line to potentially collide with the object. It can compute the interaction between a line-shaped haptic tool and the object model surface efficiently. De Novi [15] presented the Blobby modelling approach which represent organs with a set of spheres. It can perform fast collision detection between different organs and between virtual surgical instruments. Basdogan et al. [16] gave a review to survey all the important aspects of haptic device in MIS simulation, including haptic rendering, interface and data recording.

Compared with traditional surgical training method, besides providing repetitive tasks and variable complexity for trainees, VR based laparoscopic surgery simulators can also record the training progress, assess trainees’ surgical skill objectively and offering constructive feedbacks. Gurusamy et al [17] provided a total of 23 randomized clinical trials to compare virtual reality training with other forms which include no training, video training, conventional laparoscopic training for surgeons without prior laparoscopic surgery experience. 612 participants were involved in these trials. The assessment considered following factors: (1) Time, such as calculating "operating time", "time for one stitch and two ties", or "time for driving a needle through the tissue and in tying a surgical knot"; (2) Error score, such as "error score by dropping the object or perforation of object" or "average error incidence by tear of sheet, slack of ligature.”; (3) Accuracy, such as "mean number of correct incisions”; and (4) Others, such as speed, blood loss, number of movements, economy of movements, distance tracing of instruments et al. Through experimental investigation, this feedback in training can help trainees to correct surgical mistakes efficiently and make progress.

At present, most commercial medical simulators provide the basic functions of evaluation and consultancy to trainees considering a number of surgical factors, such as time of completion and wall collisions. However, for the specific laparoscopic rectum surgery training, few VR based simulators meet requirements above. In fact, the continued need for comprehensive assessment and instructor's feedback from training system is often cited as a primary reason for the reluctance of many medical schools to fully embrace virtual training technology [3]. For that purpose, we present a VR based training and assessment system for the laparoscopic rectum surgery. Firstly, referencing to the real surgery videos and MRI data, the surface mesh model of a section of rectum is constructed ad preprocessed as the training object. Generally, the cursor (proxy) from a haptic device (Phantom Omni) effects to one point during interactions. It can lead to incorrect soft tissue deformation during surgery simulation. To offer smooth and realistic geometric deformation in interactions, we have designed a generalized cylinder based collision detection algorithm for haptics. Secondly, to achieve the high deformation accuracy and real time graphic performance, we used the cosserat rod model mentioned in [18] to cope with the physical deformation of rectum. It parameterizes the centreline of the bowl 
with material coordinates which can be moved with the deforming structure. And rigid spheres are attached to the cosserat rod, which approximates the 3D shape as bounding volumes of the rectum. Thirdly, a multi-layer mass spring model is designed to cope with the deformation and surgery behaviour of membrane tissue surrounding the rectum. And a straightforward incision approach is presented based on this model. It can specially simulate the dissection procedure to detach the rectum from its surrounding tissue in real time. Finally, a hierarchy evaluation module coupled with dynamic assessment model has been implement to evalute and offer trainees constructive feedbacks in both intraoperative and post-operative manner.

\section{Materials and Methods}

\section{Operation environment and VR simulator interface}

In laparoscopic rectal resection surgery for cancer, some preoperative diagnosis and preparation should be made before operation [4]. An endoscopy is used to determine the histological confirmation of cancer and its location in the rectum. Then CT scan will be applied to provide the evidence of tumour spread to distant organs, such as lungs and liver. Sometimes, the patient will be recommended to have a MRI scan to acquire particular information on the local extent of the tumour. It can help with the formulation of a plan for treatment, in particular, whether the operation should be proceeded by chemoradiotherapy. In operation theatre, patient is usually placed in a modified Lloyd-Davis position (Fig. 1a). The number of inserted operating ports varies between surgeons, but typically 4 operating ports need be used. An operation assistant is needed to control the laparoscope and help with the retraction of tissues during operation. The surgeon relies on a variety of tools, such as stapling devices, clips and L-hooks, to help seal, grasp, dissect and separate tissues. In whole procedure, the most challenging task and eventual aim of surgery is to detach the rectum from its surrounding pelvic structure. Then the specimen will be removed from the patient through an extraction site either via a Pfannensteil incision or in the left iliac fossa [4]. Fig. 1b shows the theatre set up for the laparoscopic rectal cancer surgery.

Fig. 1c is a screenshot of our simulator interface. It illustrates a section of rectum model and the surrounding soft tissue covered. The simulator hardware (Fig. 1d) consists of a monitor, a computer and two haptic devices (Phantom Omni). Generally, one haptic device functions as a grasper, and the other functions as a L-hook or harmonic scalpel for dissection. The left haptic device can also be used as a navigation camera in this system. These haptic devices provide 6-DOF navigating parameters and 3-DOF force feedback. The software was developed with OpenGL, C++ and Qt. The overall system flow consists of three stages: preprocessing, real time operation and training evaluation. In the preprocessing, through the medical software package (3D Med 4.1) [19], the image sequence of rectum from MRI is segmented and a rough 3D rectum model is reconstructed. Referencing several real surgery videos, our animator selects a specific section of bowls and clean the model. Then some surrounding tissues are generated and attached to the rectum surface. Real time operations include the collision detection, soft tissue deformation, dissection, graphic and haptic rendering, dynamic instruction for some evaluation metrics. According to the deformation result of soft tissue, the simulator tracks the orientation and position of proxy (the tip of haptic handle) and feeds back force through haptic devices. The training evaluation module is used to record the data of the trainee, and give the score and feedback of his/her performance in or after the training. The software architecture of the system can be described as Fig. 2.

From the clinical aspect, our most important contribution is that a comprehensive framework of virtual laparoscopic rectum surgery simulation and evaluation has been provided, from system design to implementation. Compared with previous research work in VR based medical simulation, there are three innovative technical contributions as well:

1. A generalized cylinder based collision detection algorithm is presented to offer fast and efficient detection to the interaction between soft tissue and surgery instruments which has complex shape.

2. A multi-layer mass spring model is designed to cope with the deformation and surgery behaviour of membrane tissue surrounding the rectum. Correspondingly, a straightforward real-time incision approach is presented based on this model. It can specially simulate the dissection procedure to detach a section of rectum from its surrounding soft tissue.

3. A dynamic assessment model is presented and a hierarchy evaluation module has been implemented to collect and analyse the evaluation metrics for laparoscopic rectum surgery training.

\section{Generalized cylinder based collision detection for haptics}

In real time VR system, the display rate of graphic rendering is about 30 frames per second. However, in a multimodal 
virtual environment with haptic interactions, much higher update rate of about $1 \mathrm{kHz}$ is needed to ensure smooth force feedback and continuous interactions. Theoretically, the force from the haptic device (Phantom Omni) applies to only one contact point during manipulation. The most efficient interaction method is to represent the proxy (haptic cursor) as a point. However, in many cases, the interactions of instrument in surgery simulation are using long slender tools (such as dissect, shear, L-hook and so on) in which the contact surface is an area or a line. Detection problems may happen if it is simplified as a single point or a line, especially when the local concavities exist in the model (Fig. 3). In this simulator, we treat the surgery instrument as a generalized cylinder object (L-hook) or combination of multiple generalized cylinder objects (dissect, shear). And an innovative generalized cylinder based collision detection algorithm is designed. It can be described briefly as follows:

In Fig. 4, supposing the two end points of axis (dotted red line) are: $p_{0}, p_{1}$. The radius of cylinder is variable along the axis. And in a certain area the value of radius is $r$. The precondition of this collision detection algorithm is that the surgery instruments should be outside of organ objects at first when the graphic rendering starts. Then in every frame of graphic rendering loop, each vertex $(v)$ of organ object will be estimated whether it is inside of the cylinder or not. If it is inside the instrument model, the collision will take place. And a penalty force will be placed on the vertex according to its penetration depth. It can be divided into two steps:

Step 1: Calculating the angle: $\angle p_{0} p_{1} v$ and $\angle p_{1} p_{0} v$. If $\angle p_{1} p_{0} v \leq 90^{\circ}$ and $\angle p_{0} p_{1} v \leq 90^{\circ}$, then go to Step 2. If not, $v$ is outside of cylinder.

Step 2: Calculating the Euclidean distance $(d)$ between $v$ and cylinder axis line $\left(p_{0} p_{1}\right)$. If $d<r$, vertex $(v)$ penetrates the cylinder. There is a collision between tool and vertex $(v)$. And the penalty force will be placed on un

vertex $(v)$ along $v p$. Here, $p$ is the closest point on $p_{0} p_{1}$ to vertex $(v)$.

Here the radius $r$ is not a constant. It can be variable according to the shape of different area in this generalized cylinder. So it can cope with the collision detection between organ model and surgery tool which has more complex shape. Compared with other collection detection approaches, such as point-based [11, 12] and line-based [13, 14]. Generalized cylinder based collision detection algorithm has two advantages:

(1) It can solve the "Local Concavity Trap" [14]. Usually, for haptic rendering, the contact point can be trapped in the local concavities when the handle of instrument contacts with the surface of soft tissue, or the tip of the tool contacts the bottom of a deep concavity at first. This case is called "Local Concavity Trap", which can be explained by Fig. 3. In our generalized cylinder based collision detection algorithm, considered the whole shape and topology of surgery tool, each vertex on the mesh surface of soft object will be tested. Hence it prevents the "Local Concavity Trap".

(2) The "Local Concavity Trap" problem happens because only local geometry information of mesh near proximity (tip of tool), not global mesh, is considered. So to solve this problem, some modified approaches [13, 14] considered neighborhood information. They use the connection information to compute the contact triangle strip for collision detection. In our method, only the position information of model vertices is used. So it can be applied to mesh-free based model, such as point cloud.

Fig. 5 illustrates the visual performance of our collision detection algorithm compared with a standard point-based method. In Fig. 5a, the whole L-hook can touch the surface of liver and deform the model realistically. However, in Fig. 5c, the handle of L-hook is penetrated in the liver because of "Local Concavity Trap".

\section{Deformation of soft tissue}

For the colorectal surgery, large movement and deformation of soft tissues take place in the operation. It is a great challenge to compute such deformation realistically in real time. For physical-based simulation, most current approaches are based on the finite element method (FEM) [20, 21] or the mass spring system (MSS) [22, 23]. Although FEM has been widely adopted due to its good accuracy, but it may not be an effective approach for our application, when a set of volumetric mesh discretization has to be created and maintained dynamically to capture the large deformation.

In our application, two different types of deformation are involved.

(1) The actual maneuverer of the intestines can be described as slender, flexible mechanical structure when the surgical instrument interact with them. We have adopted the hybrid mechanical model developed in [18] to accurately and effectively simulate such deformation in real time, where the details is in the following section "Cosserat rectum modelling”. 
(2) In laparoscopic rectal resection surgery for cancer, the procedure often involves dissection of the fat and membrane tissues surrounding the bowl in the preparation for resection. These membrane tissues are extremely thin structures which can shrink significantly after cutting. A elastic surface model is suitable, to represent its physical behavior. We develop a multiple-layer mass spring model to simulate this behaviour which is outlined in the section of "Multi-layer mass spring model".

\section{Cosserat rectum modelling}

For a slender object, like rectum, a non-linear Cosserat rod model captures its dynamics behaviour well [24]. As in Fig. 6 , the structure of a rectum is treated as a one-dimensional geometric rod coincided with its geometric centreline. Three directors are fixed to the centreline as a metric which indicates the shearing, dilating and rotation related to the cross-section. And a dissipation energy (13) is introduced to compute the state of the rectum in interaction. Appendix A describes the detail of this model.

\section{Multi-layer mass spring model}

We use a mass spring model contained multiple layers to describe the mechanic behaviour of the membrane and fat tissues surrounding the rectum. It can be illustrated in Fig. 7 as sketch map. Here we designed four dynamic layers for this mechanic model. The first layer covers on the surface of rectum mesh. It shows as a yellow solid circle near the centre position most in Fig. 7a. The mass node on this layer can be generated by the cross-section detection of the rectum model along its media axis. The data structure of the nodes is regularized by uniform re-sampling. In our system, the data structure is treated as a $600 \times 400$ grid. The geometric position of nodes on the first layer is determined by the deformation of rectum model with the hybrid approach mentioned in (a). The fourth layer (red solid circle in Fig. 7a) is on the surface of soft tissue surrounding the rectum. The mass node can be generated by uniform re-sampling as the same manner according to the data structure of nodes on the first layer. The second layer (yellow dotted circle in Fig. 7a) is the membrane tissue attached to the rectum. The third layer (red dotted circle in Fig. 7a) is the membrane tissue attached to the soft tissue surrounding the rectum. The nodes on the second and third layer can be generated by the interpolation of the nodes pair on the first and fourth layer. And these nodes are linked in sequence by spring from the first layer to the fourth layer. The whole process above can be done automatically by following multi-layer mass spring model generation algorithm, which can be applied to all the tube like models:

Step 1: Generate the media axis of rectum model with the skeletonization algorithm presented in [26]. And the media axis is uniform sampled and constructed by an array of centre points (such as $p_{i-1}, p_{i}, p_{i+1}$ in Fig. 8a). Then, with an adjustable step length along the media axis, the cross-section of the rectum model is detected at the position of these centre points. This process can be described as Fig. 8b and Fig. 8c. Supposing the current centre point processed is $p_{i}$, we first search the nearest triangles around $p_{i}$, to detect the triangle strip which contains the silhouette of crosssection (Fig. 8b). Then the intersection points on the edges of the triangle strip with the cross-section plane through $p_{i}$ can be detected and connected in sequence as the coarse cross-section silhouette. And a fine cross-section with adjustable size of nodes can be generated after uniform re-sampling by length. Finally, every node on the current cross-section will be connected with its neighbour nodes on the previous and next cross-section by springs. After all the nodes are generated and linked as a grid $(600 \times 400)$, the first layer of multiple layers mass spring model is built.

Step 2: Generate the media axis and cross-sections of the rectum surrounding tissue. Since the soft tissue model surrounding the rectum looks like a bigger tube covered the rectum model (Fig. 7b), the approach in Step 1 can be used to generate the nodes of mass spring model for the fourth layer as the same manner. And these nodes should be aligned to their corresponding nodes on the first layer.

Step 3: Generate the nodes on the second and third layer by interpolation of the corresponding nodes pair on the first and fourth layer, and link them in sequence from the first layer to the fourth layer. The length of link between the second and the third layer is very small and variable. We treat these links as tiny springs which can be pressed or stretched during the deformation. The length of link between the first and the second layer, the third and the fourth layer is fixed. We treat these links as depth displacement from the model surface to the membrane attached.

Fig. 9 illustrates a graphic result of this multi-layer mass spring rectum model with texture in our simulator. The springs between the second and third layer are illustrated by small yellow lines (Only the springs on the first crosssection can be seen due to the occlusion). Usually, we add the parameter "Alpha" in graphic rendering for the 
membranes (the second and third layer) to show its transparency feature. For the deformation computation, we input all the nodes and links among the four layers in a single dynamic system. When this dynamic system running, for node $P_{i}$ at the time step $t$, there is:

$$
\begin{gathered}
d x_{P_{i}}(t) / d t=v_{P_{i}}(t) \\
d v_{P_{i}}(t) / d t=F_{P_{i}}(x, v, t) / m \\
F_{P_{i}}(x, v, t)=\sum F\left(P_{i}, P_{j}\right)+\sum f\left(P_{i}\right)+G\left(P_{i}\right) \\
\operatorname{uur}_{\mathbf{u r}_{i}} \operatorname{Pu}_{\dot{u_{j}}}, f\left(P_{i}\right)=-D v_{P_{i}}(t), G\left(P_{i}\right)=g m \\
\left\|P_{i} P_{j}\right\|
\end{gathered}
$$

In above equations, $X_{P_{i}}(t)$ is the $3 d$ position of node $P_{i} ; v_{P_{i}}(t)$ is the velocity of node $P_{i}$; $m$ is the mass; $F_{P_{i}}(x, v, t)$ is the sum of all the forces on $P_{i} ; F\left(P_{i}, P_{j}\right)$ is the spring force of the link between $P_{i}$ and its neighbour node $P_{j} ; L_{0}$ is the rest length of link between $P_{i}$ and $P_{j} ; \mathrm{K}$ is the stiffness of the spring; $f\left(P_{i}\right)$ is the damping force; $D$ is the damping coefficient; $G\left(P_{i}\right)$ is the gravity. We use Runge-Kutta method [27] to solve this dynamic system and set the maximum iteration time as 25. Fig. 15 (in section of "Results") gives some screenshots in tests when simulator running. The FPS (frame per second) of simulation is usually from 60 to 80 . It means the computation speed is high enough to perform a real-time graphic rendering (FPS $>30$ ). Besides the high computation efficiency, another important advantage of multi-layer mass spring model is the simplicity in dissection simulation.

\section{The simulation of dissection}

The critical procedure in laparoscopic rectal cancer surgery is cutting the tissue between the rectum and its surrounding soft tissue, to make the section of bad intestine (with tumour) disconnected with the pelvic structures and can be removed finally. So the dissection is the most important task in training. Technically, interactive realistic cutting is one of the most challenging topics in VR based surgery simulation. It has attracted a great deal of research efforts. Most cutting algorithms [28, 29, 30] based on mesh processing need to change the topology of model, even remesh the object. It is a extremely time consuming process. In this paper, inspired by the mechanism of residual strain in bio-material [31], we present a straightforward real-time incision technique which can be used to specially simulate the dissection procedure of detaching the rectum model from its surrounding tissue. This technique is designed based on the multi-layer mass spring model and can produce the realistic shrinking effect of the surrounding tissue when they are detached from the rectum.

Residual strain exists in many kinds of bio-material. It causes complexity for the estimation of deformation when a given bio-structure subjects to external loads. Experiments were designed to investigate the inner strain distribution of the residual stress but often limited to tubular structures, such as arterial [31] and oesophagus [33, 34]. Analysis by Alastrué et al. [35] showed that the incorporation of residual stress in the computation affects the circumferential stress field of arterial structure as well as the deformed configuration. In our simulation, we want to produce the shrinking effect of connective tissues surrounding the rectum near the pelvis floor when they are separated from the rectum. Such deformation could have been caused by the residual strain. In the real surgery, the surgeon has to estimate its effect before and during the dissection to reduce the risk of unwanted damage and complication. There lacks of experimental evidence to provide information for the residual strain distribution within the area of interest. The shrinking effect of rectum surrounding tissue in the real surgery video suggests that the connective tissue may subject to pre-stressed tensile force. The experiments on oesophagus [33, 34] suggested the residual strain can vary from 0 to 0.3 . Here we would assume a residual tensile strain of 0.1 uniformly distributed in the connective tissue. Prime [36] suggested the contour of the exposed surface due to a cut can be related to adding a counterpart force to the cutting plane according to the superposition principle. Therefore, given the known residual tensile stress, we can identify the deformed shape by applying the equivalent force on the surface (Fig. 10). We computed the residual stress $\sigma_{\mathrm{r}}$ as

$$
\sigma_{\mathrm{r}}=E \varepsilon_{r}
$$


Where $E$ is the Young's modulus and $\varepsilon_{r}$ is the residual strain. Here coupled with (3) and (5), we simplify the simulation of dissection as a "spring cutting" procedure, which can be illustrated as Fig. 11. The L-hook is usually used as the dissection instrument. If the tip of L-hook touches a incorrect place or vulnerable area which should be avoided in training, the system will give an alarm. Otherwise, when the tip of L-hook touches the area of the second and third layer of this model, it will search the springs between these two layers in a small sphere range (the small circle in dotted line). If a spring is detected, it will be "cut off" (deleted) from this mass spring model and removed from the dynamic system. Meanwhile, a residual tensile stress is added on the nodes linked by this spring. So with (3) and (5), there is:

$$
F_{P_{i}}(x, v, t)=\sum F\left(P_{i}, P_{j}\right)+\sum f\left(P_{i}\right)+G\left(P_{i}\right)+\sigma_{\mathrm{r}}
$$

After the computation of this updated dynamic system, a shrinking effect for the rectum and its surrounding tissue will be performed. Fig. 16 gives some experimental results of dissection simulation. Due to the residual tensile stress and the shrinking effect, we can found the rectum and its surrounding tissue are separated clearly after dissection (Fig. 16b).

\section{Evaluation for the training}

Besides providing the realistic surgical simulation in real time, our simulator can also assess trainees' surgical skills and offer trainees constructive feedback in and after simulation.

At present, several commercial medical simulators [5, 6, 7] have been developed to provide the basic functions of evaluation and consultancy to trainees. However, for the laparoscopic rectum surgery training, few VR based simulators implement the assessment functions. In reality, the continued need for comprehensive assessment and instructor's feedback from training system is often cited as a primary reason for the reluctance of many medical schools to fully embrace VR based training techniques [3]. For that purpose, to implement the assessment function for laparoscopic rectum surgery in our simulator, we define the dynamic assessment model and present a hierarchy evaluation module.

\section{Dynamic assessment model}

In the evaluation process, there are seven essential factors: evaluation subject, evaluation object, purpose, metrics, method, result and time. In our simulator, evaluation subject is the VR based laparoscopic rectum surgery simulator, which is authorized to evaluate the trainee's surgical skills; evaluation object is the trainees; the main purpose is providing constructive feedback to trainees for skill promotion through; Metrics represent aspects of surgical skills and can be composed as a hierarchy structure (Table 1) in our system for comprehensive evaluation; For the method, we apply the Fuzzy logic [37] to the data recorded, and determine the levels of expertise for each trainee considering the most major evaluation metrics. By recording the training data of virtual operation on the simulator, the evaluation result is provided to trainees after analysis. Meanwhile, during the procedure of simulation, the system can also instruct the trainees when improper manipulation happens. For example, the system will give alarm when the trainee grasping or cutting a vulnerable area which should be avoided. So the evaluation exists in intra-operative and postoperative intervention procedures. Based on the descriptions above, we define and build a dynamic assessment model, which is shown in Fig. 12. In this figure, the evaluation results can give feedback to the trainees to improve their training behaviour in real time. So this process forms a dynamic loop.

\section{Hierarchy Evaluation Module}

The comprehensive evaluation of surgical skills is difficult since it involves the assessment of all aspects of surgeon's techniques, such as the ability of correct decision making in response to the discrete events in a surgical scenario, maintaining the proper visibility of the surgical field, achieving sufficient exposure of critical anatomy structures, removing the optimal volume of tissue, apply appropriate velocities and forces when vulnerable anatomy structures are approached, et al. It is not practical to consider all the possible factors to assess a trainee. And some factors belong to the domain of psychology, which is hard to be measured by a quantitative approach. So we should select the key evaluation metrics which fit the given surgery best, and assemble these metrics to a form which can reflect the trainee's skill in a higher level. Here, compared with some existing simulators [5, 7], we set up a comprehensive evaluation module with hierarchy structure rather than providing the basic evaluation metrics only.

The surgical skill hierarchy evaluation module covers the most major training targets with different hierarchy levels. Its structure can be illustrated as Table 1. In this table, evaluation metrics are divided into four grades: the first grade indicates the surgical expertise of the trainee. The other three grades are composed by subsets of concrete evaluation metrics. On the second grade, the surgical skills are evaluated by following three categories of metrics: Efficiency (the 
time spent on training task, and the quantity of redundant fabrics used), Safety (the parameters that measure the safety of operations in virtual training, such as the ability to avoid unwanted collisions between instruments and vulnerable tissues), Quality (the parameters which measure the performed skills of trainees in the task). We also define the adjustable weights for each evaluation metrics, which are illustrated in Fig. 13. After the weights setting, the system can compute the total score for trainees. The personal information and training record for each individual will be listed below the Window of “Learning Centre” in the system interface (Fig. 14a). After all the trainees' performance are evaluated and recorded, the system will classify the trainees to different levels according to the evaluation scores of their surgical expertise. And the distribution of level for all the trainees can be illustrated in the system interface (Fig. 14b).

\section{Results}

Implementing the techniques above, a prototype virtual reality based simulation and assessment system is developed for the laparoscopic rectum surgery. When the system starts, the simulator generates a continuous loop with force feedback. The haptic devices send the position and orientation information of surgery instruments to the computer. The collisions detection between the surgery instruments and organ models are processed according to these data. The soft tissue deformation and the feedback force are computed by the hybrid mechanical model of rectum and the multilayer mass spring model of rectum surrounding tissue. Then the signal of feedback force will be sent to the haptic devices for rendering. When simulator running, the assessment module will record all the evaluation metrics for each trainee. Meanwhile, since the assessment is a dynamic process, it can give trainee instructions in real time when improper manipulation happens. Finally, after the training finished, the system will give a score to each trainee and illustrates statistical data for this trainee group. Our system is running on a Workstation with Intel CoreQuad 2.83GHz, 6 GB RAM and equipped with a NVIDIA Quadro FX 3700 graphics card. The force refreshing frequency is $1000 \mathrm{~Hz}$ and the graphic refreshing frequency is $45 \mathrm{~Hz}$. The total cost of the system hardware is about 12,000 US dollars, which is approximately $1 / 6$ of the cost to purchase a commercial VR surgery simulator (etc. Lap Mentor [6]).

To verify the effectiveness of the techniques presented in our system, we test the dissection simulation which is the most important training task in the surgery. In this test, trainee use three instruments (one L-hook and two graspers) to detach a section of rectum from its surrounding soft tissue. From the experimental results illustrated in Fig. 15, we can found the deformation is plausible. After dissection, the rectum and its surrounding tissue are separated clearly. Moreover, our simulator also implement special visuals, such as smoking, for the training (Fig. 15f).

We also let ten subjects with varying laparoscopic colorectal surgery experience tested and assessed our system. The subjects were asked to perform the procedure of dissection (Fig. 16) on our simulator, and then to answer a feedback questionnaire consisting of nine questions: (1) realism of the anatomy, (2) realism of the appearance of the simulator interface, (3) realism of the instrument handling, (4) overall realism of the dissection task, (5) quality of force feedback in performing the tasks (6) usefulness in learning hand-eye coordination (7) usefulness in learning ambidexterity skills, (8) overall usefulness in learning the fundamental surgical skills (9) Accuracy of the evaluation for the performance in training. Each question can be answered on a 5 point Likert scale with 5 for very good satisfactory and 1 for very poor. The results from the questionnaire study are given in Table 2 .

\section{Discussion}

The first VR based surgery simulator for rectum cancer is designed. Throughout the whole development process for this simulator, three senior surgeons from the Royal Bournemouth and Christchurch Hospital, Poole Hospital (NHS, UK) have been involved in this project closely. They advise us about the medical content and assist to evaluate the training results as both consultants and trainees. A satisfactory tactile feedback is achieved finally according to their operation experience. Through haptic rendering, users can watch the realistic internal surgery environment with visual outputs and tactile feeling in real time.

In the evaluation process, the result from the preliminary study (Table 2) shows the average scores for all the questions. It also indicates which aspects need to be improved for more realistic and useful simulation. In Table 2, the lowest score (3.1) are "the realism of the anatomy". Due to the computation complexity, we currently modify the original rough rectum model to a clean and smooth one. In future, we can apply GPGPU programming (e.g. CUDA) to enhance the graphic computation power for our system. Then we can choose more complex rectum model for surgery simulation. 


\section{Conclusion and future work}

We have developed a prototype virtual reality based training and assessment system for the laparoscopic rectum surgery. Several technical innovations have been presented in this article, including the generalized cylinder based collision detection, the multi-layer mass spring model for the deformation and rectum dissection, a dynamic model for hierarchy evaluation. And real-time visual performance are achieved. The surgeon collaborators are satisfied with the realistic visual quality and tactile feedback of our simulator.

Despite successful implementation of the prototype, this simulator also has a few limitations. Since Phantom Omni provides only a 3-DOF force feedback, the torque is not considered during the surgery training process. In real laparoscopic surgery, the proper cutting torque is required. So we plan to choose more sophisticated haptic devices coupled with torque feedbacks to enhance the tactile realism for dissection simulation. We also plan to add GPGPU programming to enhance its graphic computation speed further for our system. Then we can choose more complex rectum model for surgery simulation. Furthermore, currently we just implement the function of training assessment. In future, more trials and study will be made in the assessment of laparoscopic rectum surgery training through our system. With the collected and analyzed data, this VR based training system will be compared quantitatively with other approaches, such as physical based training and standard laparoscopic training.

\section{Acknowledgements}

The research in this article was supported by a grant from the Royal Bournemouth and Christchurch Hospitals NHS Trust and a HEIF grant from Bournemouth University.

\section{References}

1. Ali MR, Mowery Y, Kaplan B, DeMaria EJ. Training the novice in laparoscopy. More challenge is better. Surgical Endoscope 2002; 16 (12):1732-1736

2. Aggarwal R, Moorthy K, Darzi A. Laparoscopic skills training and assessment. British Journal of Surgery 2004; 91(12):1549-1558.

3. Grantcharov TP. Virtual reality simulation in training and assessment of laparoscopic skills. European Clinics in Obstetrics and Gynaecology 2006; 2( 4) : 197-200 .

4. Francone T, Weiser MR. Laparoscopic Rectal Resection for Cancer. Seminars in Colon and Rectal Surgery 2005; 16(3): 147-154.

5. http://www.surgical-science.com/portfolio/lapsim-basic-skills/

6. http://simbionix.com/simulators/lap-mentor/

7. http://www.mentice.com/

8. Pan J, Chang J, Yang X, Qureshi T, Howell R, Hickish T, and Zhang J. Graphic and haptic simulation system for virtual laparoscopic rectum surgery. International Journal of Medical Robotics and Computer Assisted Surgery 2011; 7: 304-317.

9. Choi C, Kim J, Han H, Ahn B, Kim J. Graphic and haptic modeling of the oesophagus for VR based medical simulation. The International Journal of Medical Robotics and Computer Assisted Surgery 2009; 5: 257-266.

10. Heng PA, Cheng CY, Wong TT, Xu YS, Chui YP, Chan M, Tso SK. A Virtual Reality Training System for Knee Arthroscopic Surgery. IEEE Trans. Information Technology in Biomedicine 2004; 8(2): 217-227.

11. Lin MC. Otaduy M. Haptic Rendering: Foundations, Algorithms and Applications, A. K. Peters, Ltd. 2008.

12. Govindaraju NK, Knott D, Jain N, Kabul I, Tamstorf R, Gayle R, Lin MC, Manocha D. Interactive collision detection between deformable models using chromatic decomposition. In ACM SIGGRAPH 2005, New York, NY, USA: 991-999.

13. Chih-Hao H, Basdogan C, Srinivasan MA. Ray-based haptic rendering: Force and torque interactions between a line probe and 3D objects in virtual environments. International Journal of Robotic Research 2000; 19(7): 668-683.

14. Maciel A, De S. An efficient dynamic point algorithm for line-based collision detection in real time virtual environments involving haptics. Comput. Anim. Virtual Worlds 2008; 19 (2): 151-163.

15. Dott. Ing. Gianluca De Novi., Soft tissue modeling for virtual reality surgery simulator with haptic feedback ( $\mathrm{PhD}$ thesis)

16. Basdogan C, De S. Haptics in Minimally Invasive Surgical Simulation and Training. IEEE Computer Graphics 2004; 24(2): 56-64.

17. Gurusamy KS, Aggarwal R, Palanivelu L, Davidson BR. Virtual reality training for surgical trainees in laparoscopic surgery. Cochrane Database System Review 2009; 1 (1).

18. Chang J, Yang X, Pan J, Li W, and Zhang J. A fast hybrid computation model for rectum deformation. The Visual Computer 2010; 27: 97-107.

19. http://www.mitk.net/

20. Bathe KJ. Finite Element Procedures, 2nd edition, London: Prentice-Hall, 1996.

21. Wu W, Heng PA. A hybrid condensed finite element model with GPU acceleration for interactive 3D soft tissue cutting. Computer Animation and Virtual Worlds 2004; 15(3-4): 219-227.

22. Mollemans W, Schutyser F, Cleynenbreugel J, Suetens P. Fast soft tissue deformation with tetrahedral mass spring model for maxillofacial surgery planning systems. MICCAI 2004; Springer-Verlag, Berlin, Heidelberg. 3217: 371-379. 
23. Hong M, Jung S, Choi M, Welch SW. Fast volume preservation for a mass-spring system. IEEE Computer Graphics and Application 2009; 26(5): 83-91.

24. Antman, SS. Nonlinear Problems of Elasticity, Applied Mathematical Sciences. Vol. 107, Springer Verlag. 1995.

25. Chang J, Yang X, Zhang JJ. Continuous Skeleton-driven Skinning - A General Approach for Modelling Skin Deformation. International Journal of Image and Graphics 2009; 9(4): 591-608.

26. Pan J, Yang X, Xie X, Willis P, Zhang JJ. Automatic rigging for animation characters with 3D silhouette; Computer Animation and Virtual Worlds 2009; 20 (2-3): 121-131.

27. Atkinson, Kendall A. An Introduction to Numerical Analysis (2nd Ed. 1989), New York: John Wiley \& Sons.

28. Ganovelli F, O'Sullivan C. Animating cuts with on-the-fly re-meshing. Eurographics 2001; Manchester, UK. $243-247$.

29. Picinbono G, Lombardo JC, Delingette H, Ayache N. Improving Realism of a Surgery Simulator: Linear Anisotropic Elasticity, Complex Interactions and Force Extrapolation. J. Visual. Comput. Animat 2002; 13: 147-167.

30. Cotin S, Delingette H, Ayache N. A hybrid elastic model for real-time cutting, deformations, and force feedback for surgery training and simulation. The Visual Computer 2000; 16(8): 437-452.

31. Chang J, Zhang JJ. Force mapping. In: Theory and Practice of Computer Graphics 2004, Bournemouth, U.K., 204-210.

32. Chuong CJ, Fung YC. On residual stress in arteries. Journal of Biomechanical Engineering 1986; 108(2): $186-192$.

33. Lu X, Gregersen H, Regional distribution of axial strain and circumferential residual strain in the layered rabbit oesophagus. Journal of Biomechanics 2001; 34(2): 225-233.

34. Zhao J, Chen X, Yang J, Liao D, Gregersen H. Opening angle and residual strain in a three-layered model of pig oesophagus. Journal of Biomechanics 2007; 40(14): 3187-3192.

35. Alastrué V, Garía A, Peña E, Rodríguez JF, Martínez MA, Doblaré M. Numerical framework for patient-specific computational modelling of vascular tissue. International Journal for Numerical Methods in Biomedical Engineering 2010; 26(1): 35-51.

36. Prime MB. Cross-sectional mapping of residual stresses by measuring the surface contour after a cut. Journal of Engineering Materials and Technology 2001; 123(2): 162-168.

37. Hájek P. Fuzzy logic and arithmetical hierarchy. Fuzzy Sets and Systems 1995; 3(8): 359-363

\section{Appendix A: Non-linear Cosserat rod model}

By convenience, the three directors $\left(\mathbf{d}_{1}, \mathbf{d}_{2}, \mathbf{d}_{3}\right)$ are chosen as an orthogonal framework at a given location $\mathbf{r}\left(\boldsymbol{x}_{1}, \boldsymbol{x}_{2}, \boldsymbol{x}_{3}\right)$ and the third director $\mathbf{d}_{3}$ is chosen to be along the tangent of the beam centreline. The Darboux vector $\boldsymbol{\omega}$ is defined as

$$
\frac{\partial \mathbf{d}_{i}}{\partial s}=\boldsymbol{\omega} \times \mathbf{d}_{i}, i=1,2,3 \text {; }
$$

which includes the torsion $(\tau)$ and the flexures $\left(\kappa_{1}, \kappa_{2}\right)$ related to the deformation:

$$
\boldsymbol{\omega}=\kappa_{1} \mathbf{d}_{1}+\kappa_{2} \mathbf{d}_{2}+\tau \mathbf{d}_{3}
$$

Using the Langrangian approach, we can formulate the motion equations as

$$
\frac{d}{d t}\left(\frac{\partial T}{\partial \mathbf{q}_{k}}\right)-\frac{\partial T}{\partial \mathbf{q}_{k}}+\frac{\partial U}{\partial \mathbf{q}_{k}}+\frac{\partial D}{\partial q_{k}}=\int \mathbf{J}_{k} \mathbf{f} d s
$$

$T$ is the kinetic energy, $U$ is the potential energy, $D$ is the dissipation energy. $\mathbf{J}_{k}$ is the Jacobian matr $\mathbf{J}_{k}=\partial \mathbf{r} / \partial \mathbf{q}_{k}$, and $\mathbf{f}$ is the external forces.

$$
T=\frac{1}{2} \int \rho A \frac{d \mathbf{r}}{d t} \cdot \frac{d \mathbf{r}}{d t} d s
$$

The kinetic energy $T$ is defined by the velocity of the beam's centreline and its angular part is ignored, where $\rho$ is the mass density and $A$ is the cross-sectional area.

$$
U=\frac{1}{2} \int\left[B_{1}\left(\kappa_{1}-\bar{\kappa}_{1}\right)^{2}+B_{2}\left(\kappa_{2}-\bar{\kappa}_{2}\right)^{2}+G J(\tau-\bar{\tau})^{2}+E A\left(v_{3}-1\right)\right] d s
$$


The potential energy $U$ is defined as elastic energy that induced by bending (the $1^{\text {st }}$ and $2^{\text {nd }}$ terms in the integration), twisting (the $3^{\text {rd }}$ term in the integration) and the elongation (the $4^{\text {th }}$ term in the integration) parts. $v_{3}=\frac{\partial \mathbf{r}}{\partial s} \cdot \mathbf{d}_{3}$ represents the length change along the tangent direction. Values with bar above, correspond to the rest configuration of the beam without any external forces, which can be viewed as residual items. $B_{1}=E I_{1}$ and $B_{2}=E I_{2}$ are the bending stiffness of the beam, where $E$ is the Young's modulus, $I_{1}$ and $I_{2}$ are the respective area moment of inertia for the cross section. $G$ is the shear modulus and $J$ is the polar moment of inertia for the cross section.

$$
D=\frac{1}{2} \int\left[\gamma_{1}\left(\frac{d \kappa_{1}}{d t}\right)^{2}+\gamma_{2}\left(\frac{d \kappa_{2}}{d t}\right)^{2}+\gamma_{3}\left(\frac{d \tau}{d t}\right)^{2}\right] d s
$$

The dissipation energy $\mathrm{D}$ is related to the non-conservative forces and $\gamma_{i}$ are the damp coefficients.

In equation (13), $\mathbf{q}_{k}$ is the general displacement vector and $\underset{k}{\&}$ is the general velocity vector of the $k$-th node. In our discretization, $\mathbf{q}_{k}$ are the angular values which describe the orientation change of local frame defined by directors in Fig. 6a plus the local elongation parameter at the $k$-th node position. Equation (13) leads to the equation of motion expressed by the general displacement vector $\mathbf{q}_{k}$ :

$$
M \mathbb{q}_{k}+C \mathbf{q}_{k}+K \mathbf{q}_{k}=\mathbf{R}
$$

where $q_{k}$, is the acceleration of $\mathbf{q}_{k}$, and $\&_{k}$ is the velocity. $M$ is the general mass matrix, $C$ is the damp matrix and $K$ is the stiffness matrix. $\mathbf{R}$ is the residual part which is written as $\mathbf{R}=K \overline{\mathbf{q}}_{k}+\int \mathbf{J}_{k} \mathbf{f}_{k} d s$. We solve the equation (13) to update the state of the rectum in interaction. For details of collision handling, readers can refer to $[8,18]$ 


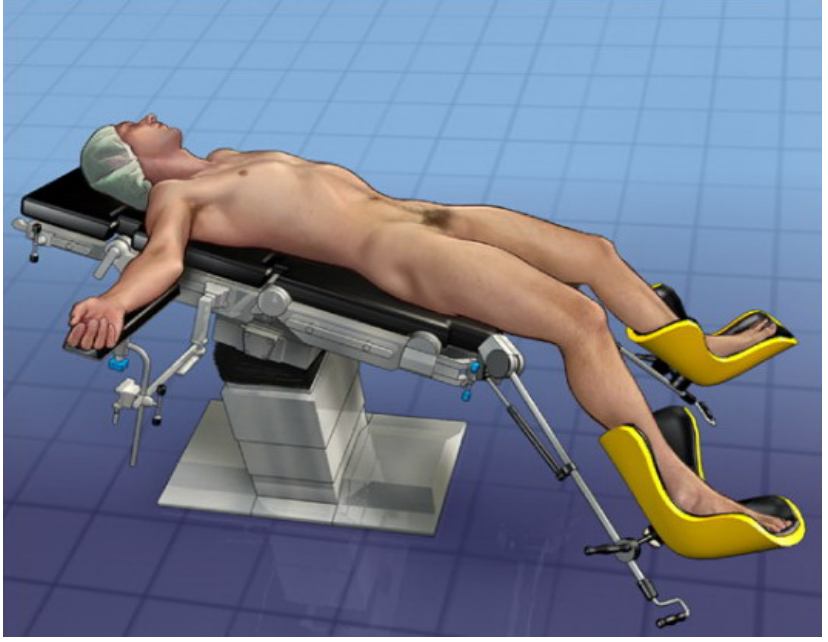

(a)

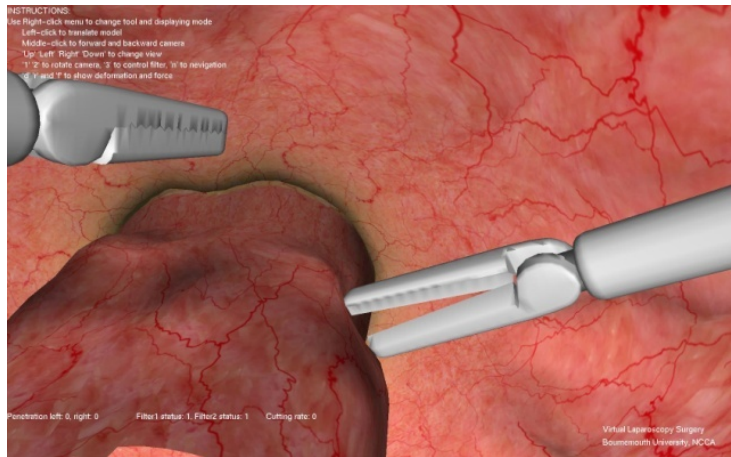

(c)

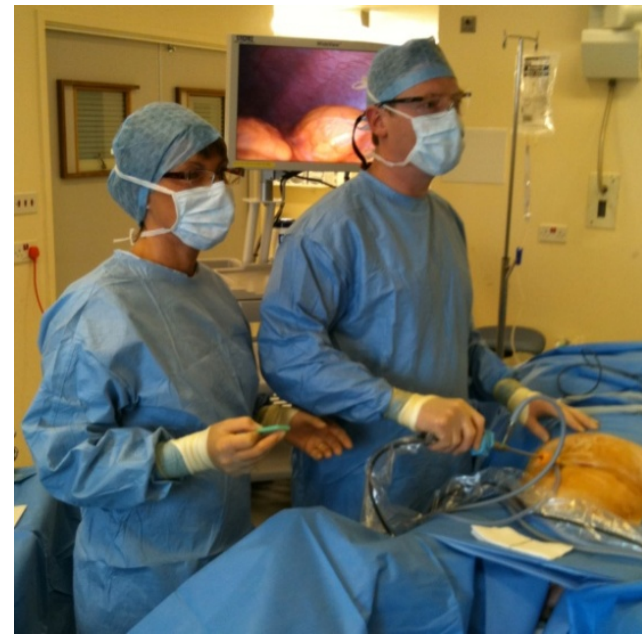

(b)

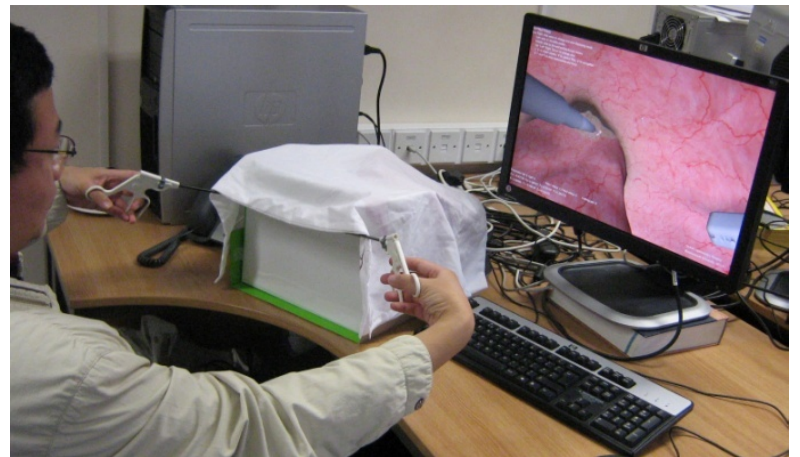

(d)

Fig. 1. Comparison of real laparoscopic rectum surgery with our VR based simulation system. (a) Modified Lloyd Davies. (b) Real operation environment for the laparoscopic rectum surgery. (c) The software interface of our VR based simulator. (d) Snapshot of our VR based simulator hardware. 


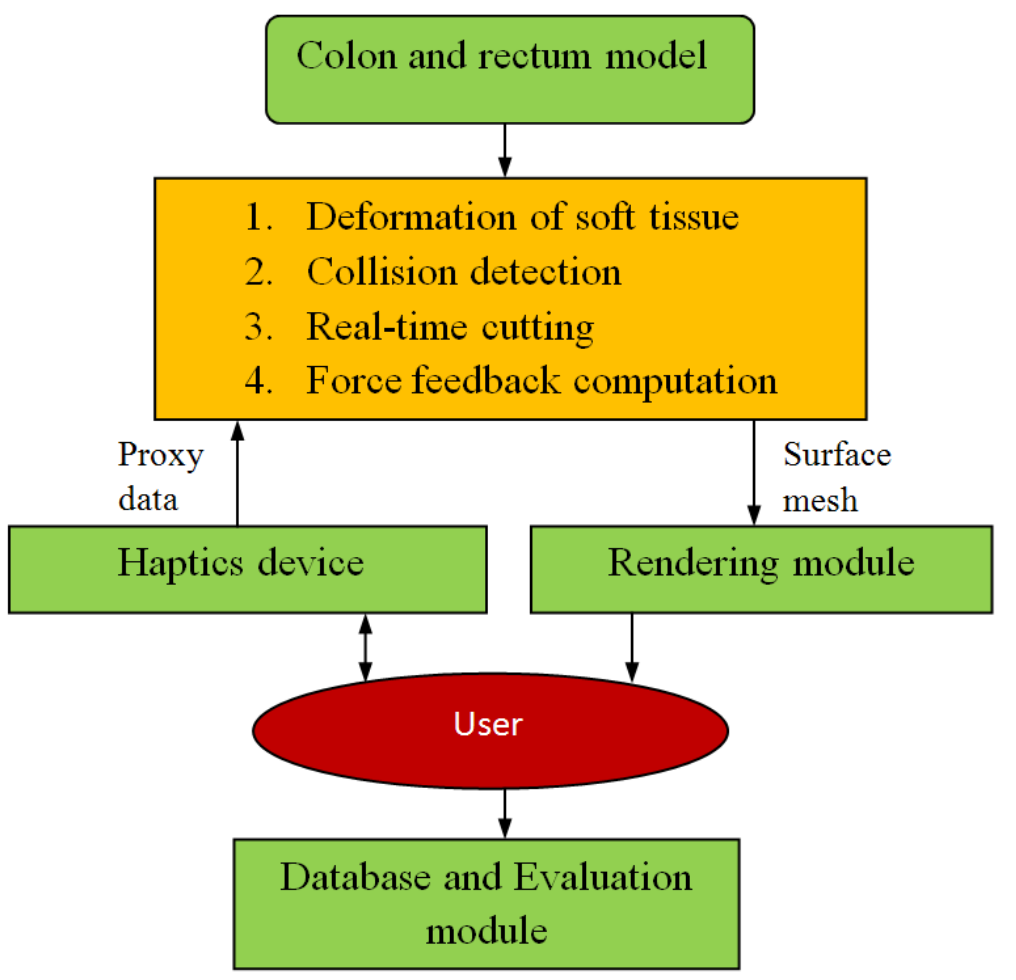

Fig. 2. The system architecture of the VR based laparoscopic rectum surgery simulator 


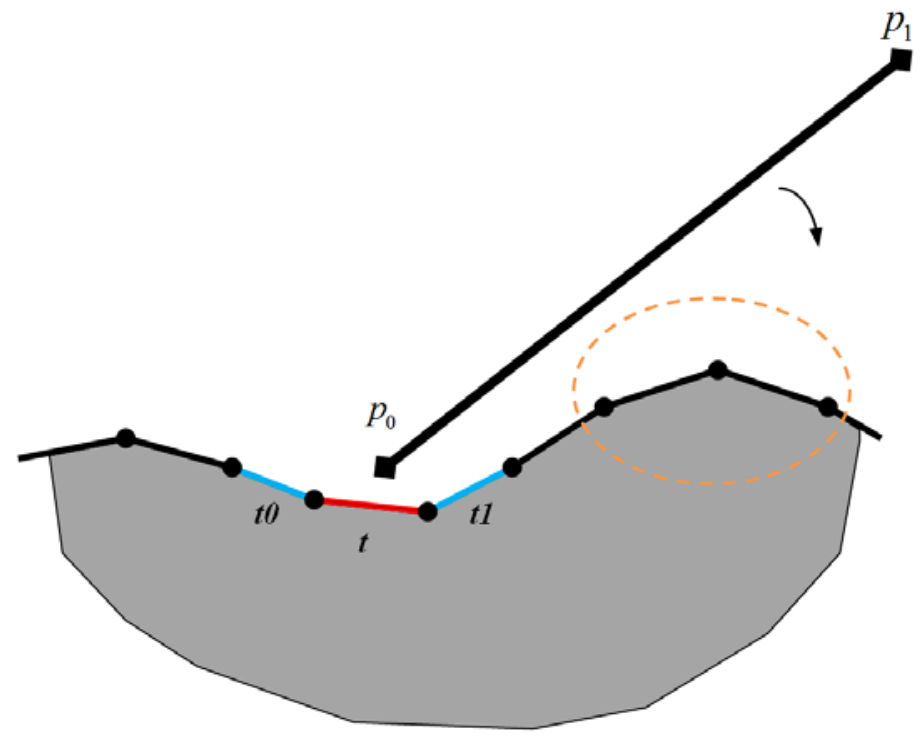

(a)

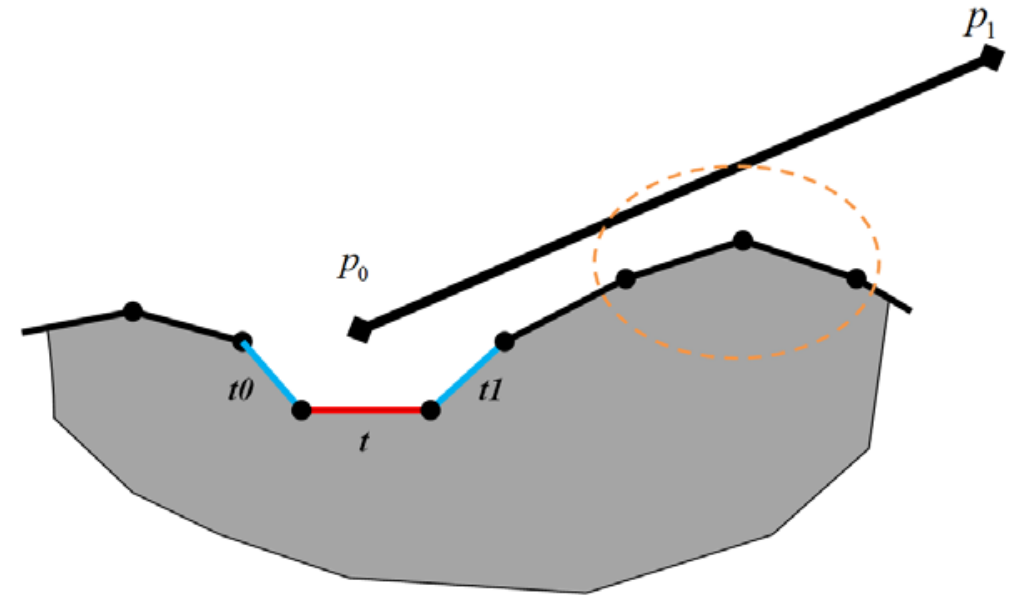

(b)

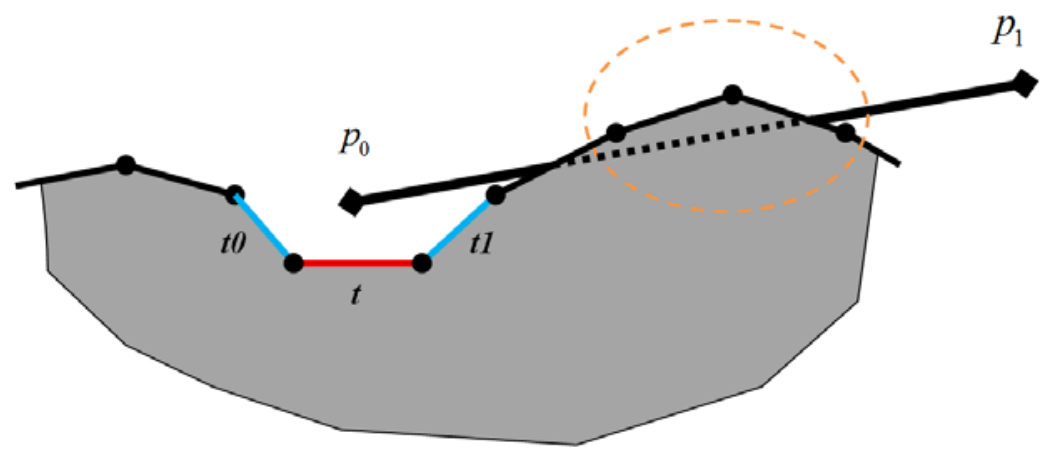

(c)

Fig. 3. Small concavity can trap the line-based tool to a local region when it interacting with deformable objects. (a) A line cursor collides with the surface of soft object, (b) Deformed surface, (c) Triangles around the small crest area (yellow circle) eventually penetrate the line-based tool 


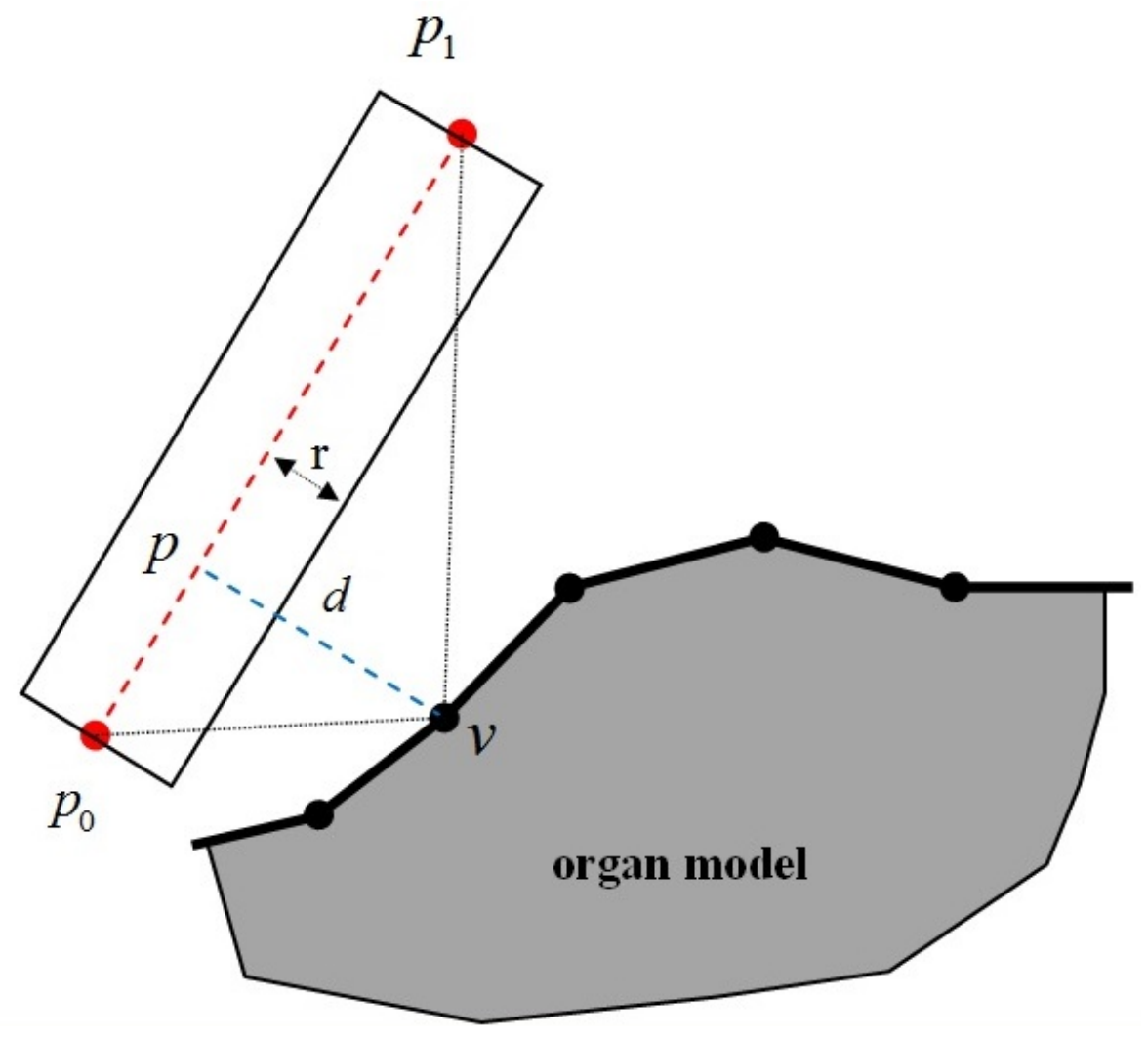

Fig. 4. Generalized cylinder based collision detection 


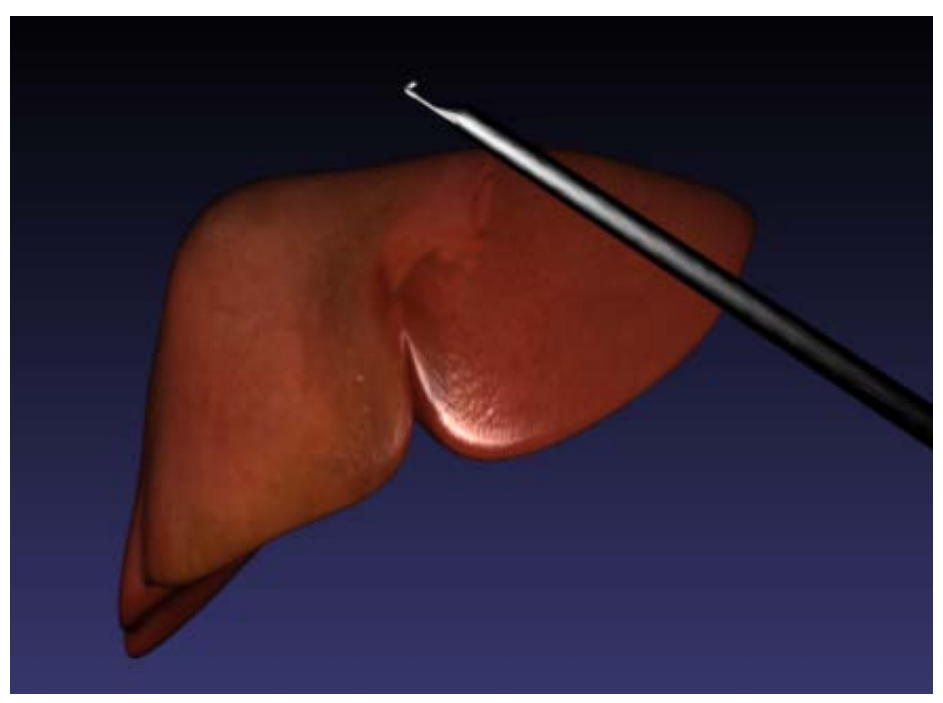

(a)

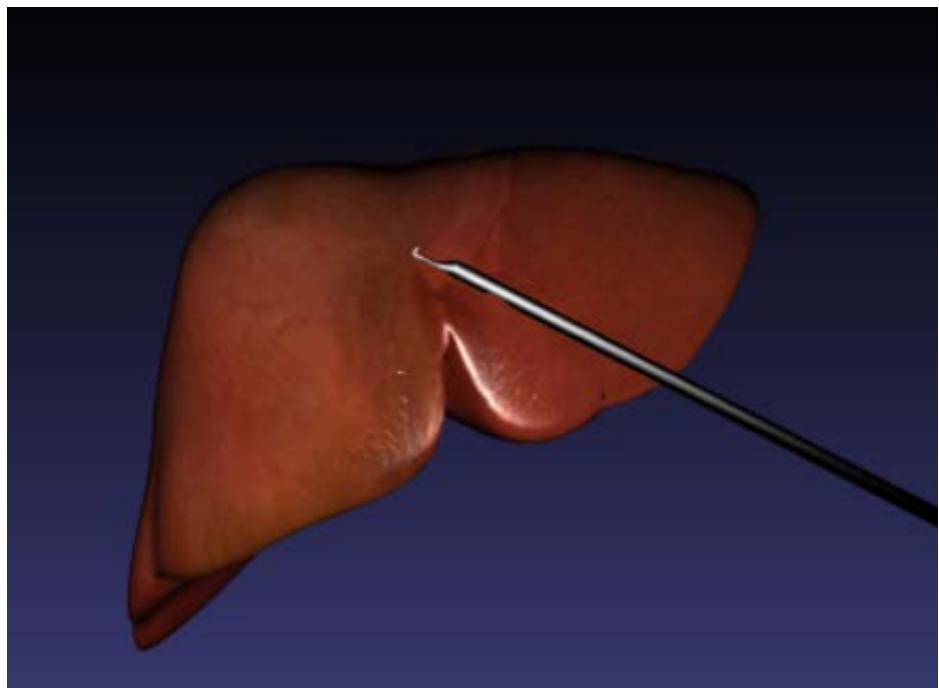

(b)

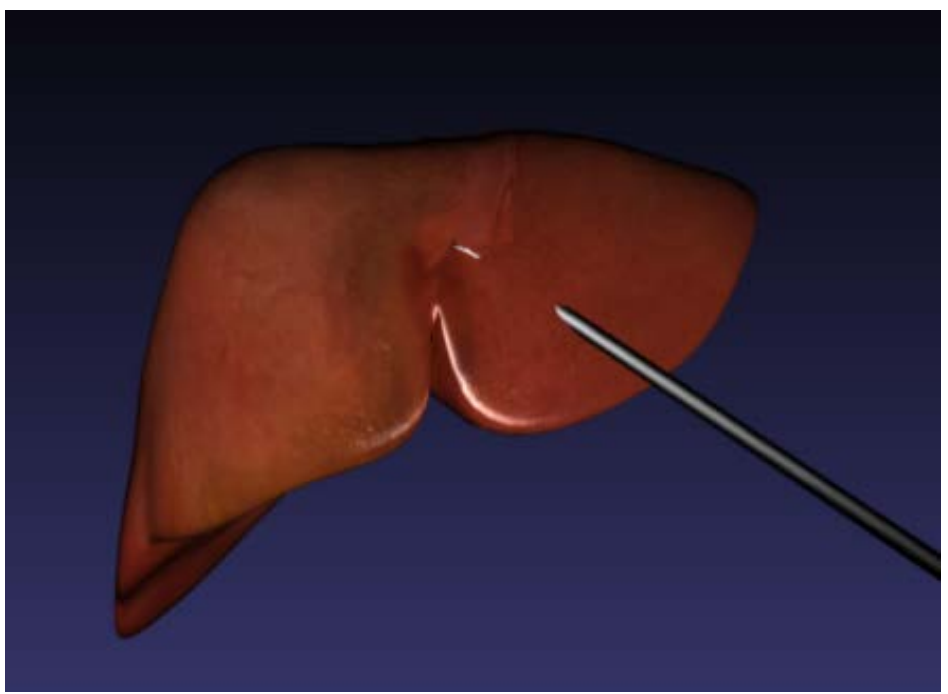

(c)

Fig. 5. The comparison of visual performance of a L- hook touching the surface of liver model. (a) Before collision, (b) Collision detection by our generalized cylinder based algorithm, (c) Collision detection by a point-based method 


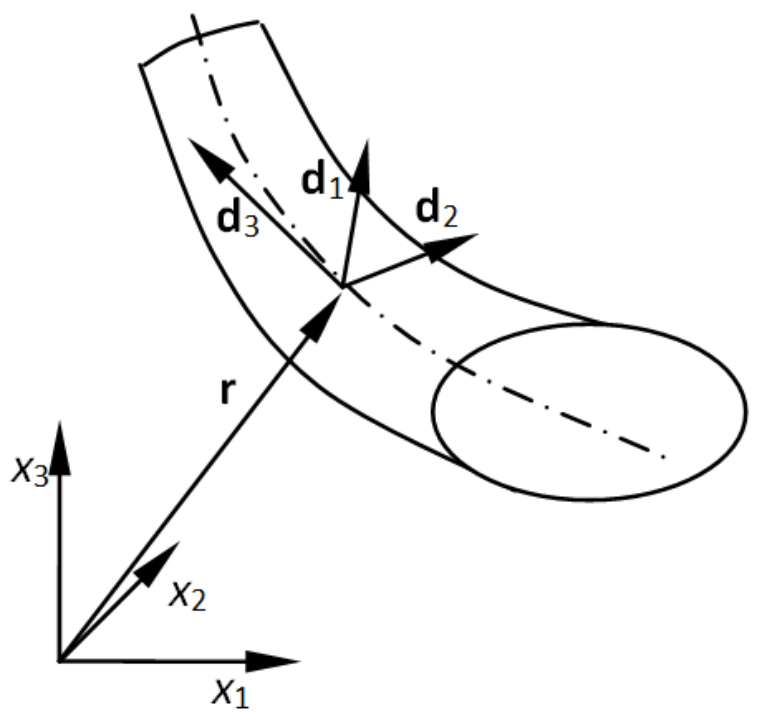

(a)

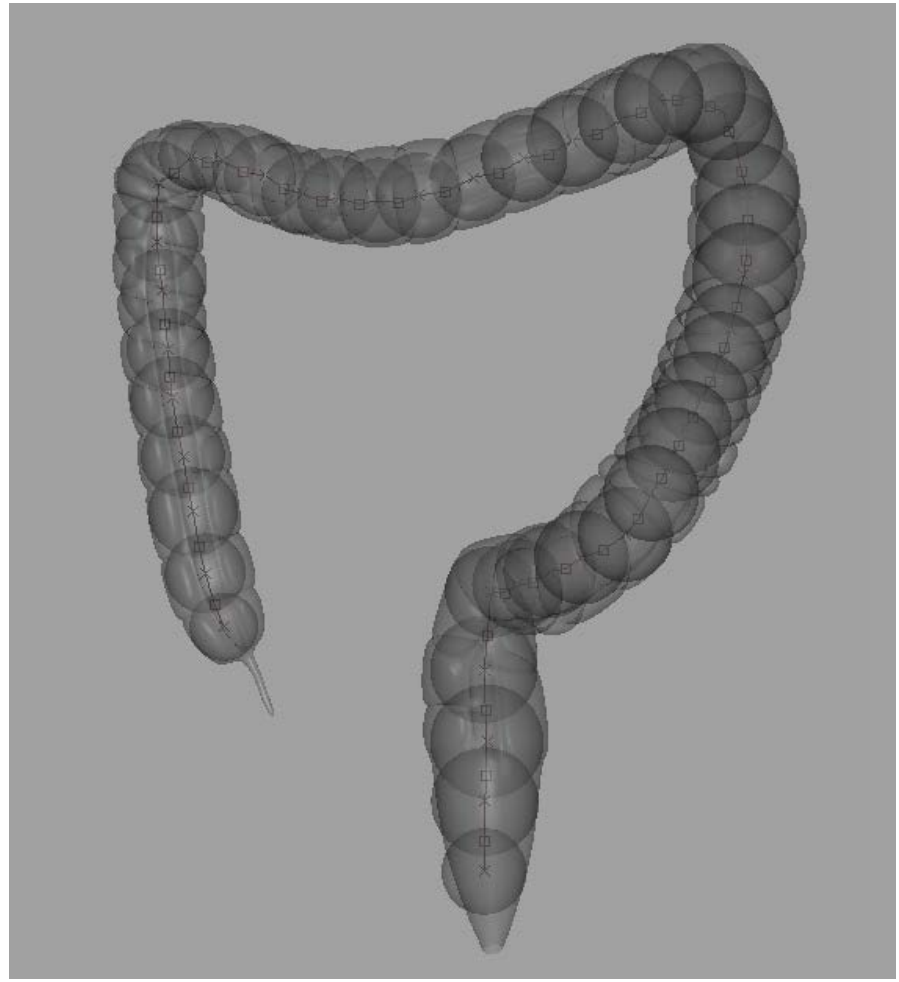

(b)

Fig. 6. Cosserat rod model of rectum. (a) Without losing generality, a 2D diagram is given, (b) A section of intestine with Crosserat rod model 


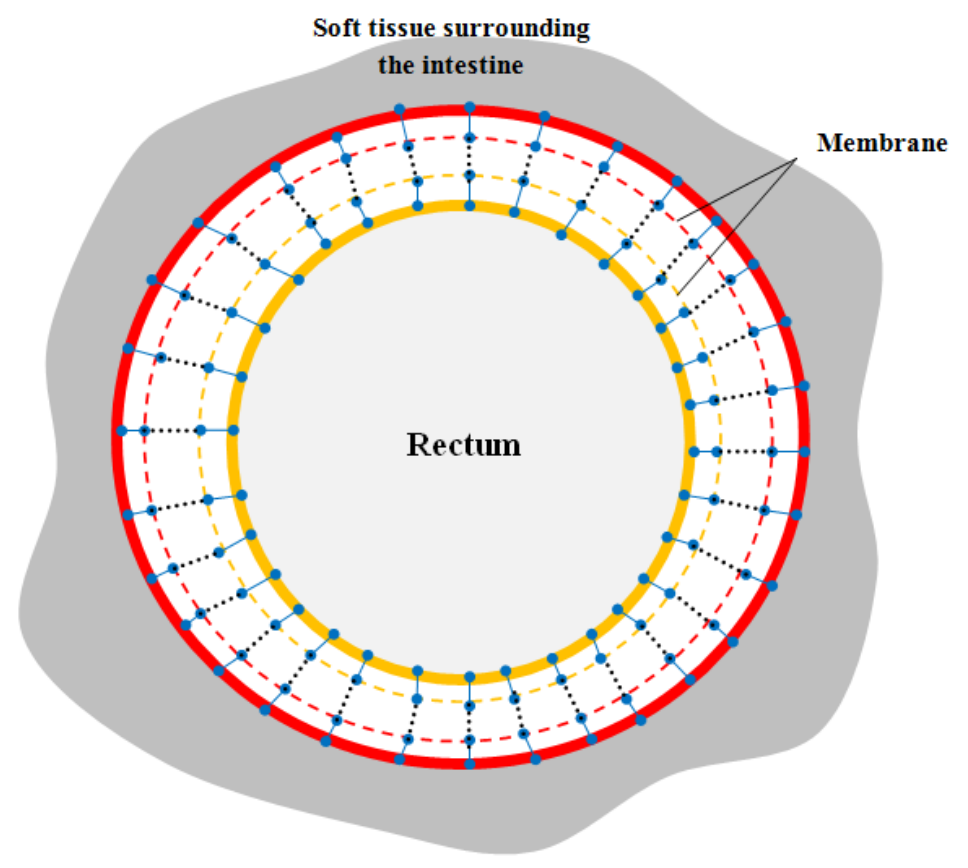

(a)

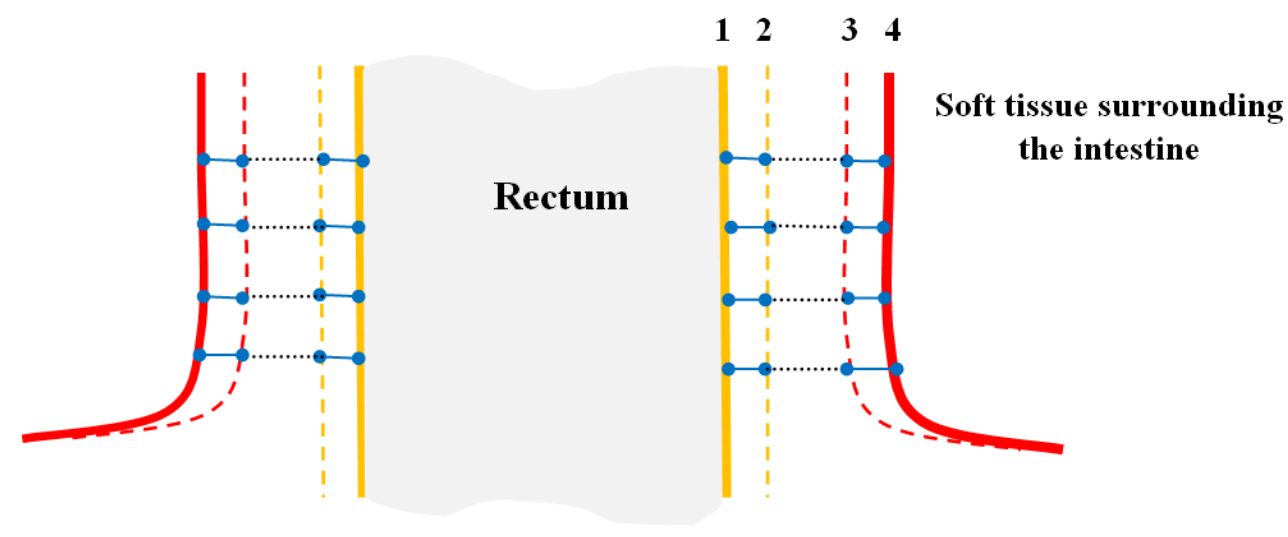

(b)

Fig. 7. "Multi-layer mass spring model" for the membrane and fat tissues surrounding the rectum. (a) The sketch map on the cross section, (b) The side profile 


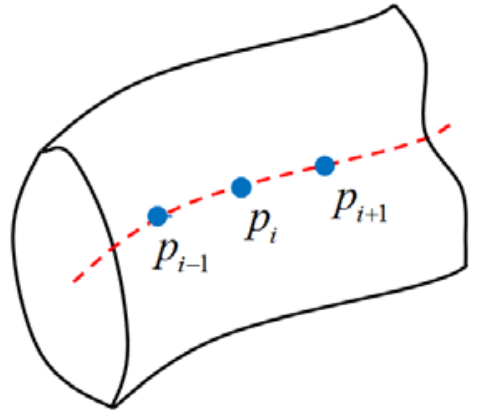

(a)

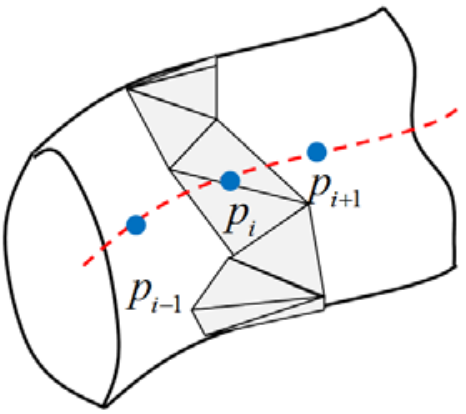

(b)

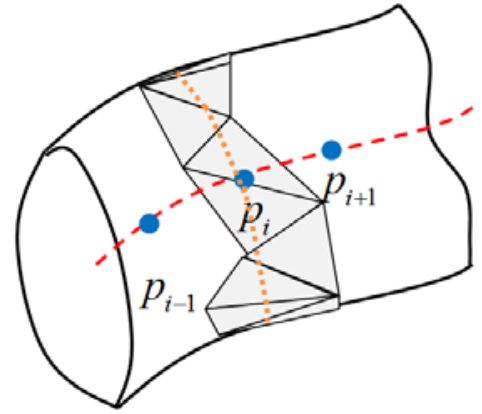

(c)

Fig. 8. The generation of media axis and the cross-section nodes along the axis. (a) Media axis for a section of intestine, (b) Triangle strip around $p_{i}$, (c) Cross-section with adjustable number of nodes around $p_{i}$ 


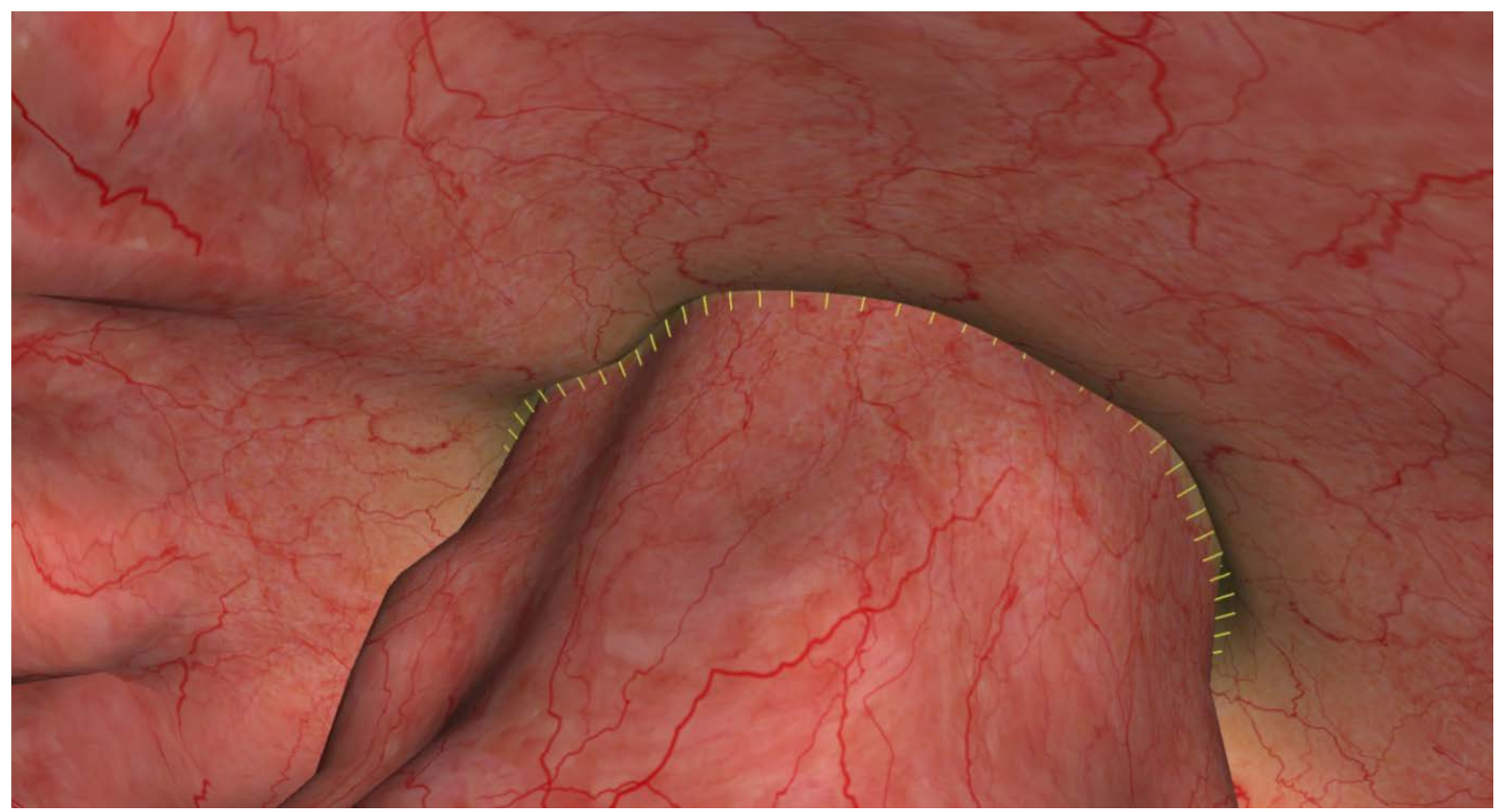

Fig. 9. The multi-layer mass spring rectum model with texture in our simulator (yellow lines stand for the springs between the second and third layer) 

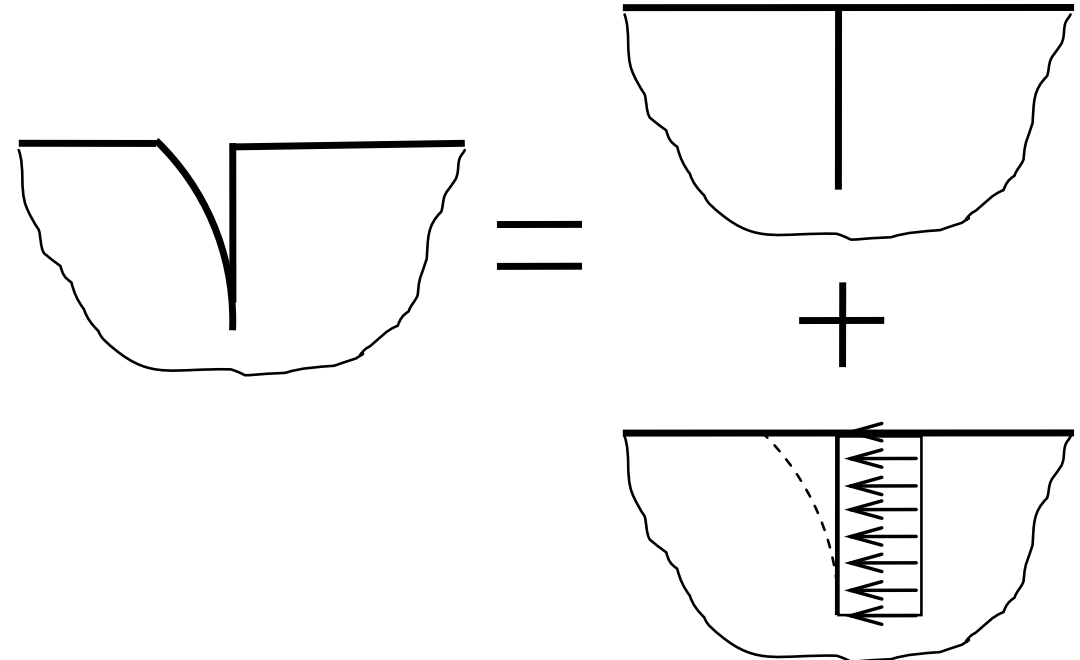

Fig. 10. The deformation caused by the residual stress is equivalent to the results of a straight cut followed by equivalent force applied on the cutting surface 


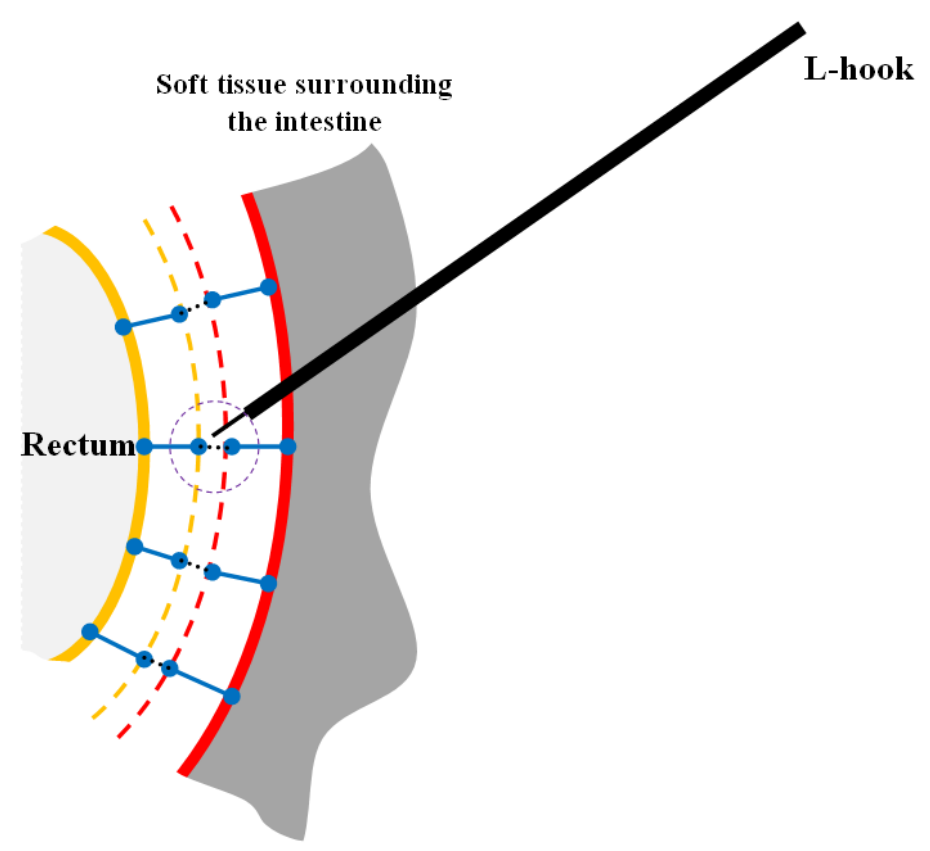

(a)

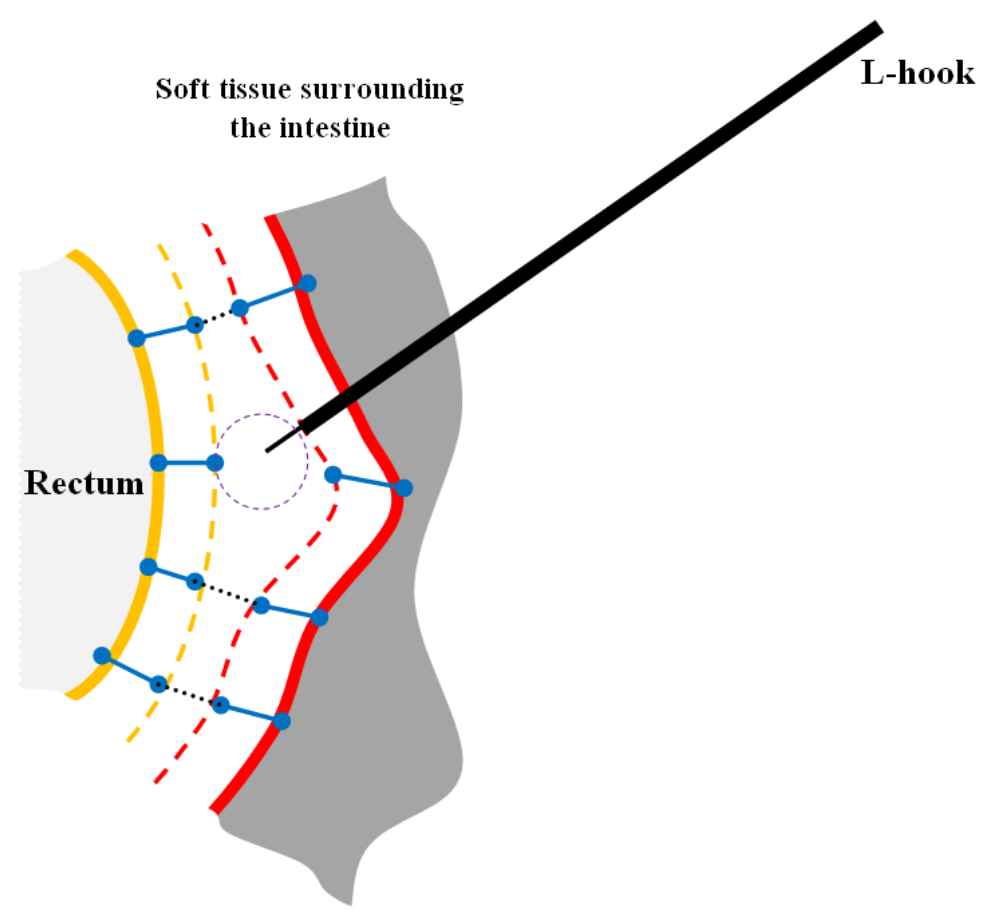

(b)

Fig. 11. The simulation of dissection to separate the rectum and its surrounding soft tissue. (a) Before cutting the spring, (b) After cutting the spring 
Table 1. The metrics in hierarchy evaluation module for our simulator

\begin{tabular}{|c|c|c|c|}
\hline First Grade & Second Grade & Third Grade & Fourth Grade \\
\hline \multirow{16}{*}{ Surgical Skill } & \multirow{2}{*}{ Efficiency } & Completion Time & \\
\hline & & Redundant Fabric Used & \\
\hline & \multirow{6}{*}{ Safety } & \multirow{3}{*}{ Exposure } & Directly Exposed \\
\hline & & & Indirectly Exposed \\
\hline & & & Overexposed \\
\hline & & Wall Collision Times & \\
\hline & & Near Vulnerable Tissue Forces & \\
\hline & & Near Vulnerable Tissue Velocities & \\
\hline & \multirow{8}{*}{ Quality } & \multirow{6}{*}{ Drilling \& Cutting Technique } & Cutting Length \\
\hline & & & Cutting Depth \\
\hline & & & Cutting Region \\
\hline & & & Cutting Angle \\
\hline & & & Jump Frequency \\
\hline & & & Instrument Selection \\
\hline & & \multirow{2}{*}{ Accurate Technique } & Ratio of Correct Cutting, \\
\hline & & & Ratio of Incorrect Cutting \\
\hline
\end{tabular}




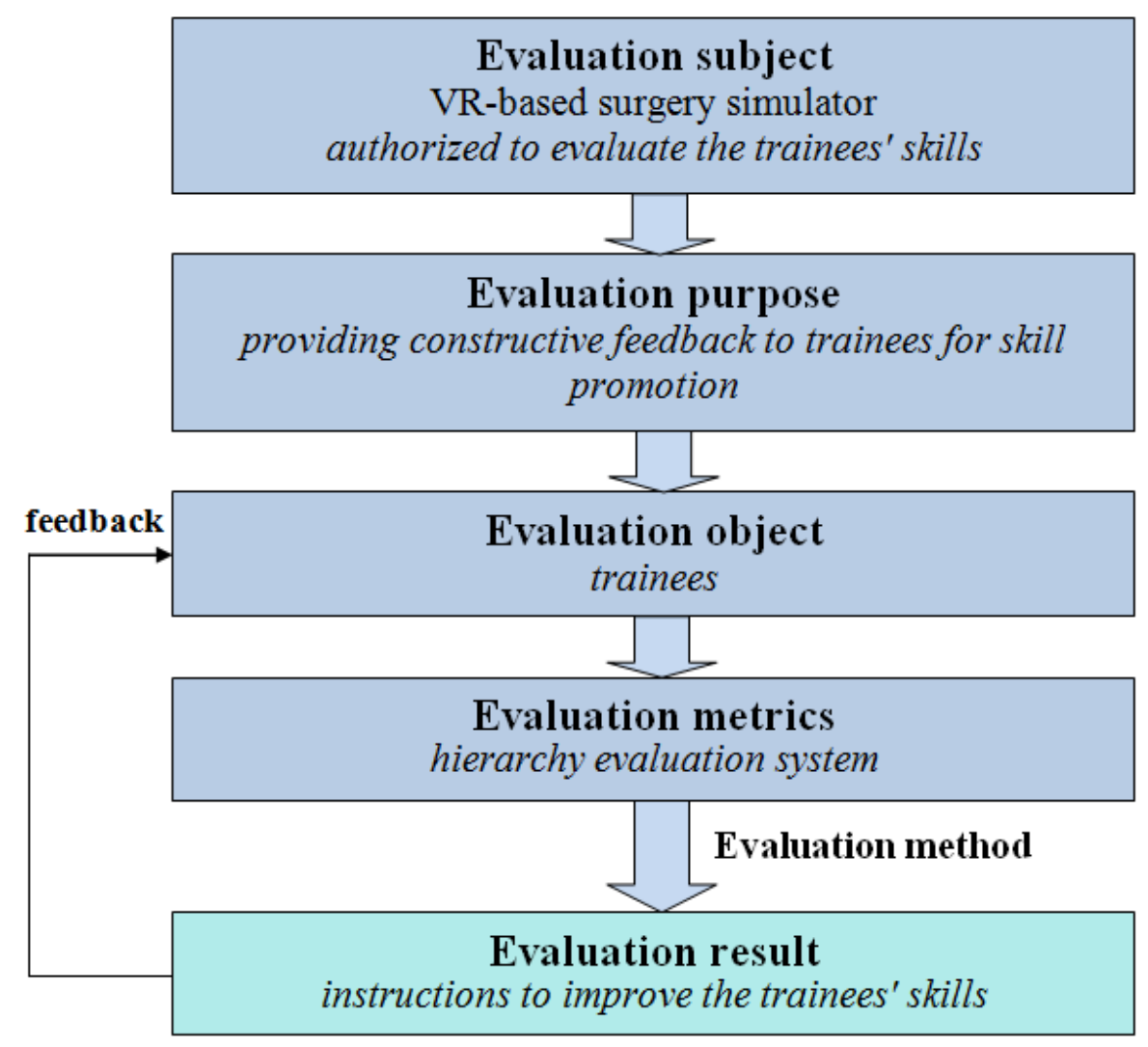

Fig. 12. Dynamic assessment model 

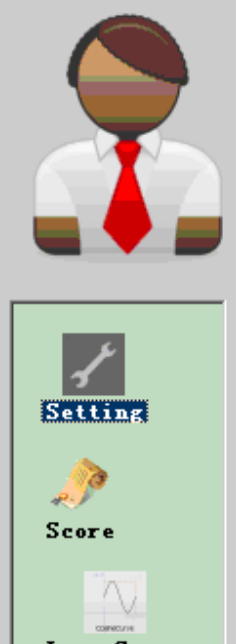

Le arnCurve

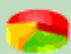

Statistics

(i)

Fffectractor

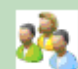

Userlanage

Weight Setting

I Basic Skill

HO FLY ZONE $0.01 \div \sqrt{1} \sim \sqrt{44}$ Collision Duration $\quad 0.04 \div \sqrt{4} \sim \sqrt{33}$

Unreached $\quad 0.04 \div \sqrt{2} \sim \sqrt{22}$ Incorrect Remove Ratio $0.04 \div \sqrt{1} \sim \sqrt{31}$

Collision Times $0.04 \div \sqrt{3} \sim \sqrt{33}$ Correct Remove Ratio $0.04 \div \sqrt{2} \sim \sqrt{31}$ II Advance Skill

I Safety Skill

Exposure $\quad 0.04 \div \sqrt{3} \sim \sqrt{44}$ Vulnerable Force $\quad 0.04 \div \sqrt{2} \sim 5$ Out of Focus $\quad 0.04 \div \sqrt{4} \sim \sqrt{41}$ Vulnerable Velocity $0.04 \div \sqrt{3} \sim 5$ View Obscured(sec) $0.04 \div \sqrt{1} \sim \sqrt{5}$

II Efficiency Skill

Completion Time $0.04 \div \sqrt{2} \sim \sqrt{25}$ Instruments Selection $0.04 \div \sqrt{3} \sim \sqrt{33}$

Optimized route $0.04 \div \sqrt{2} \sim \sqrt{55}$ Instruments Usage $\quad 0.04 \div \sqrt{3} \sim \sqrt{22}$ Moving Efficiency $0.04 \div \longdiv { 3 } \sim 2$

III Quality Skill

Incision Length $0.07 \div \sqrt{3} \sim \sqrt{33}$ Force

Incision Depth $0.04 \div \sqrt{3} \sim \sqrt{5}$ Jump Frequency

$0.04 \div \sqrt{4} \sim 6$

Incision Region $0.03 \div \sqrt{1} \sim \sqrt{6}$ ScalpelaSuction Distance $0.04 \div \sqrt{4} \sim 55$

Incision Angle $0.05 \div \sqrt{3} \sim \sqrt{6}$ Executive dust $\quad 0.04 \div \sqrt{5} \sim \sqrt{6}$

Incision Duration $0.04 \div 3$

Fig. 13. Weight setting for evaluation metrics 


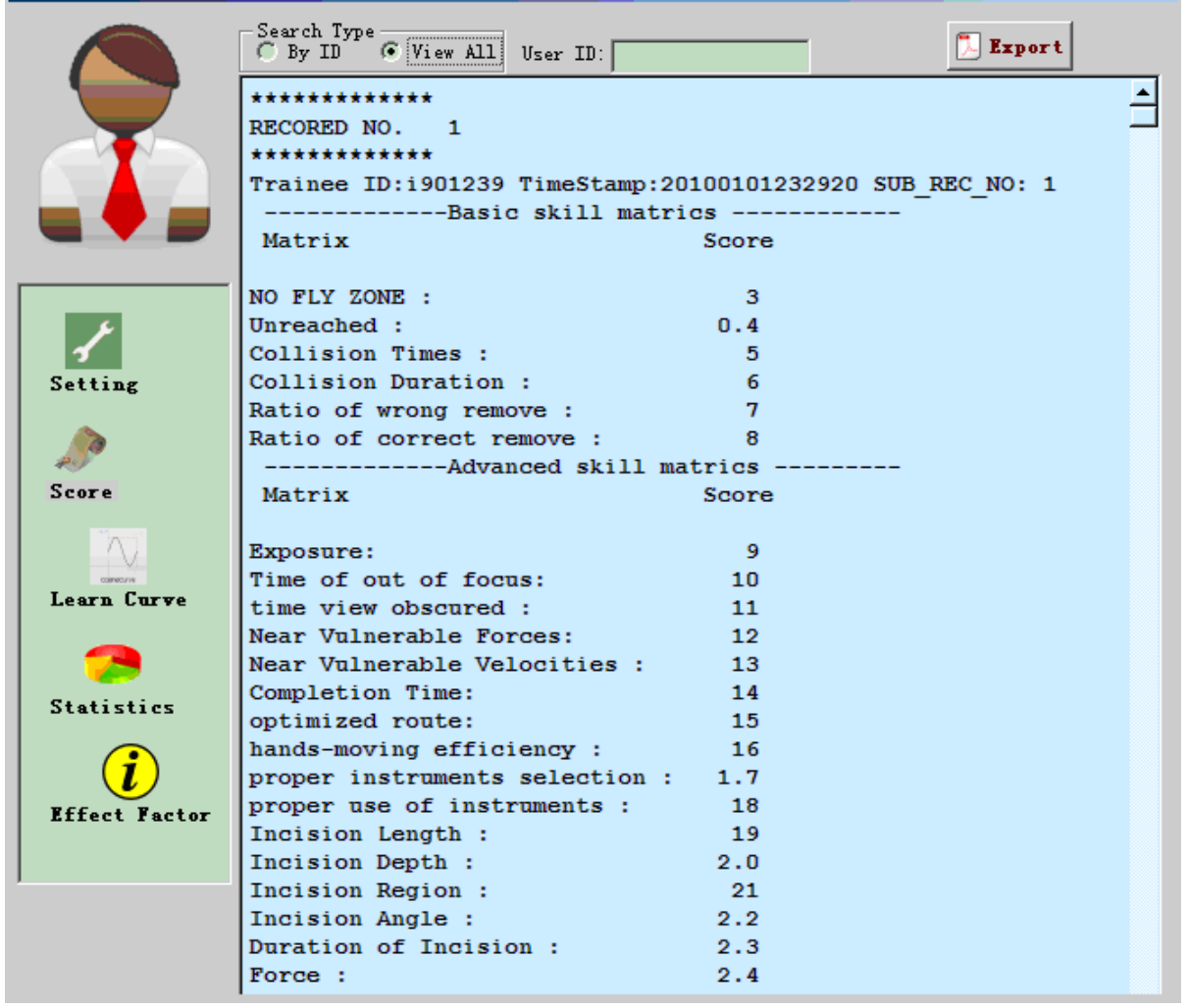

(a)

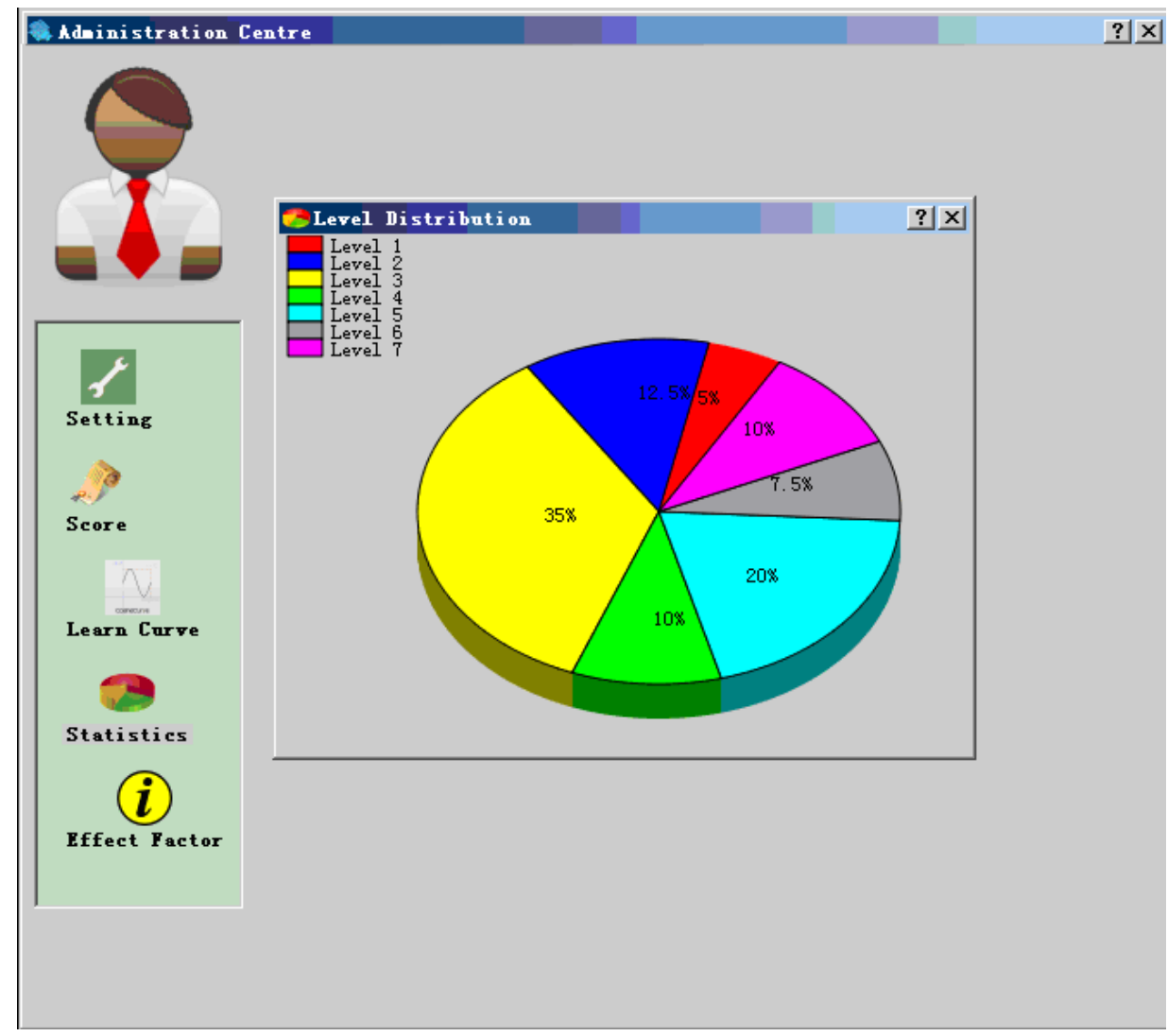

(b)

Fig. 14. The evaluation results in the software interface of our simulator. (a) Recorded training data and score, (b) The distribution of evaluaed surgical skill levels for all trainees 


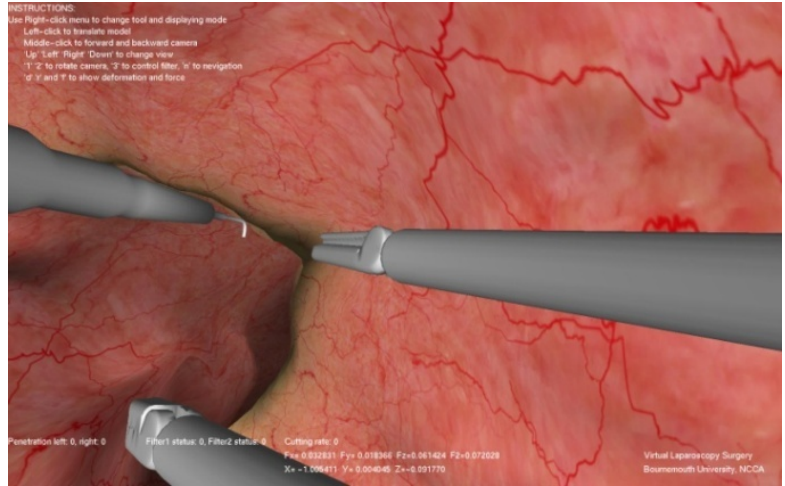

(a)

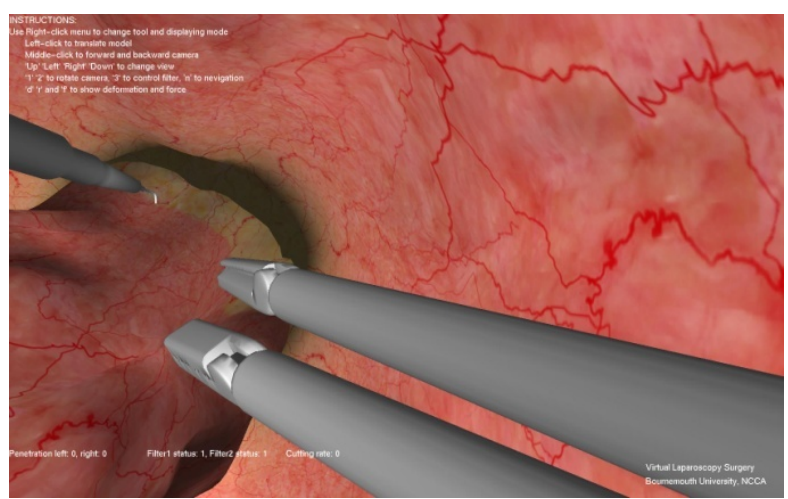

(c)

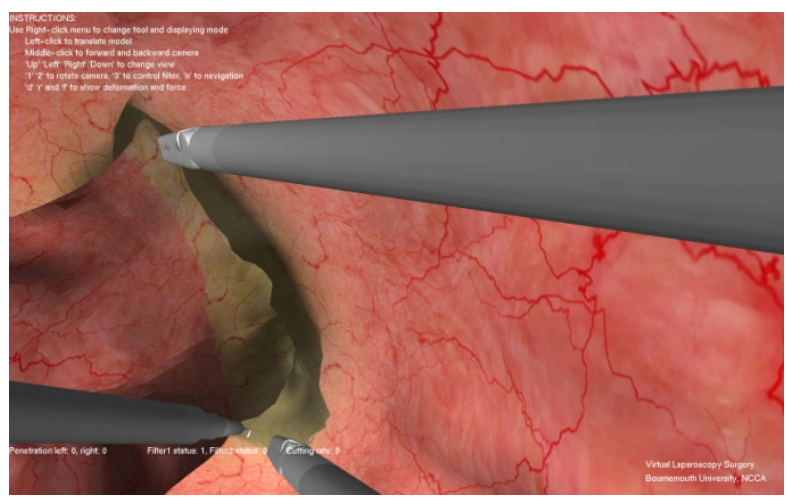

(e)

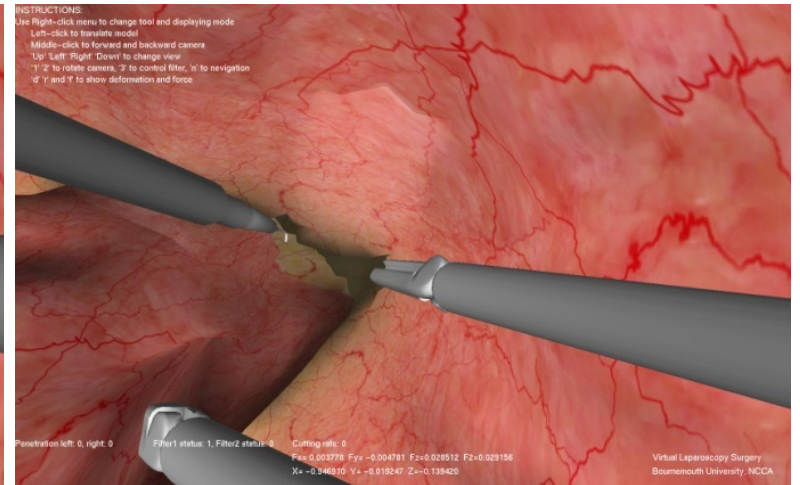

(b)

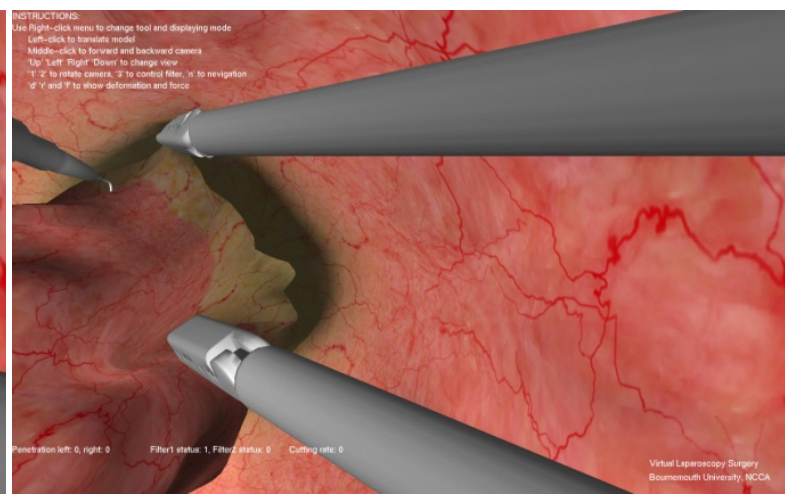

(d)

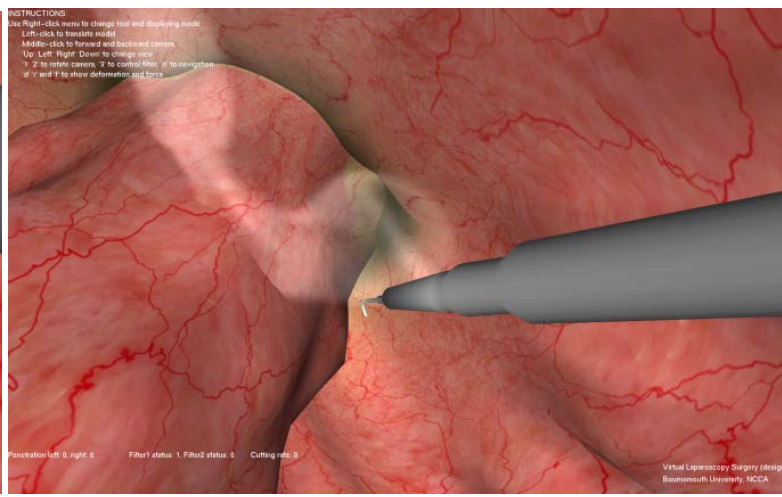

(f)

Fig. 15. The screenshots of dissection to detach the rectum from its surrounding tissue. (a) Before dissection, (b) 5 seconds after dissection procedure starts, (c) 60 seconds after dissection procedure starts, (d) 80 seconds after dissection procedure starts, (e) 100 seconds after dissection procedure starts, (f) smoking effect during dissection simulation 
Table 2. Descriptive statistics obtained from the questionnaire study

\begin{tabular}{|c|c|c|c|c|c|c|c|c|c|}
\hline & 1 & 2 & 3 & 4 & 5 & 6 & 7 & 8 & 9 \\
\hline Mean & 3.1 & 3.5 & 3.4 & 3.6 & 3.9 & 3.8 & 3.7 & 4.1 & 3.4 \\
\hline SD & 0.88 & 0.97 & 0.84 & 1.17 & 0.82 & 0.78 & 0.99 & 0.87 & 0.99 \\
\hline
\end{tabular}

